\title{
An extended equation of state for core-collapse simulations
}

\author{
M. Oertel, ${ }^{1}$ A. F. Fantina, ${ }^{2}$ and J. Novak $^{1}$ \\ ${ }^{1}$ Laboratoire Univers et Théories, CNRS/Observatoire de Paris/Université \\ Paris Diderot, 5 place Jules Janssen, 92195 Meudon, France \\ ${ }^{2}$ Institut d'Astronomie et d'Astrophysique, CP-226, \\ Université Libre de Bruxelles, 1050 Brussels, Belgium
}

(Dated: October 29, 2018)

\begin{abstract}
In stellar core-collapse events matter is heated and compressed to densities above nuclear matter saturation density. For progenitors stars with masses above about 25 solar masses, which eventually form a black hole, the temperatures and densities reached during the collapse are so high that a traditional description in terms of electrons, nuclei, and nucleons is no longer adequate. We present here an improved equation of state which contains in addition pions and hyperons. They become abundant in the high temperature and density regime. We study the different constraints on such an equation of state, coming from both hyperonic data and observations of neutron star properties. In order to test the zero-temperature versions, we perform numerical simulations of the collapse of a neutron star with such additional particles to a black hole. We discuss the influence of the additional particles on the thermodynamic properties within the hot versions of the equation of state and we show that in regimes relevant to core-collapse and black hole formation, the effects of pions and hyperons on pressure, internal energy and sound speed are not negligible.
\end{abstract}

PACS numbers: 26.60.Kp,26.50.+x

\section{INTRODUCTION}

Supernovae and hypernovae figure among the most spectacular events observed in the universe because of the immense amount of energy involved. In general, one can distinguish between thermonuclear and core-collapse events. Here we shall be interested in the latter. These occur at the end of the life of massive $\left(M \gtrsim 8 M_{\odot}\right)$ stars: when the iron core exceeds the Chandrasekhar mass a gravitational collapse is induced. At the center a compact star is formed, which is a neutron star in the classical gravitational supernova. Depending among others on the progenitor mass, its metalicity, and rotation, as well a black hole can be formed. These events are known as hypernovae or collapsars. For about thirty years, simulations have been performed in order to explore these events and to answer related questions, for example on the precise conditions for forming a neutron star or a black hole. The simulations are extremely complex, since they involve many different ingredients: multidimensional hydrodynamics, neutrino transport, general relativity and complicated microphysics. Despite all the effort, many unknowns remain in the simulations, in particular on the engine driving a successful supernova explosion. Apart from the observations via electromagnetic radiation, the neutrino and gravitational wave signal could give interesting information on the models.

The microphysics input for the simulations concerns essentially two domains, the rates for neutrino-matter interaction and deleptonization, i.e. electron capture, and the equation of state (EOS). Here we will discuss the latter. It is not an obvious task to construct an EOS. The main difficulty arises from the fact that very large ranges of (baryon number) densities $\left(10^{-10} \mathrm{fm}^{-3} \lesssim n_{B} \lesssim\right.$ $\left.1 \mathrm{fm}^{-3}\right)$, temperatures $(0<T \lesssim 150 \mathrm{MeV})$ and hadronic charge fractions $\left(0<Y_{q}=n_{q} / n_{B} \lesssim 0.7\right)$ have to be covered. $n_{q}$ here denotes the total hadronic charge density, which in many cases is just given by the proton density. Within this range the characteristics of nuclear matter change dramatically, from an ideal gas of different nuclei up to uniform strongly interacting matter, containing in the simplest case just free nucleons but potentially other components such as hyperons, nuclear resonances or mesons. Even a transition to deconfined quark matter cannot be excluded. Although there is a large variety of EOSs available for cold dense matter relevant for the description of neutron stars (see for example [1] and references therein), at present, only a few hadronic EOSs exist which are commonly used in core collapse simulations, where temperature effects play a crucial role. There is the one by Hillebrandt and Wolff [2], used by some groups performing supernova simulations, that by Lattimer and Swesty [3] as well as that by H. Shen et al. [4]. The two latter, publicly available, are most commonly used in core-collapse simulations. They use different nuclear interactions, but are based on the same limiting assumptions: they take into account non-interacting $\alpha$-particles, a single heavy nucleus and free nucleons in addition to the electron, positron and photon gas.

However, in particular at low densities, i.e. below roughly nuclear matter saturation density $n_{B} \lesssim n_{0} \approx$ $0.16 \mathrm{fm}^{-3}$ (corresponding to a mass density of about $\left.\sim 10^{14} \mathrm{~g} / \mathrm{cm}^{3}\right)^{1}$ the composition of matter is much more

\footnotetext{
${ }^{1}$ We shall work here exclusively with baryon number densities, since the baryon number is a conserved quantity, notably throughout a hydrodynamic simulation, contrary to the mass density which is not conserved. Many codes, for dimensional reasons, work, however, with a mass density. The latter can be
} 
complicated, with a large number of different nuclei. Although this should not have a large impact on the purely thermodynamical properties [6], it is important to correctly describe the composition of matter in order to determine the electron capture rates and neutrino interactions. Therefore, in the last years, several groups have started to build EOSs using mainly statistical approaches to improve the low density part of the EOS (see e.g. [713]). It has been shown that especially the presence of additional light nuclei can have an influence on the supernova dynamics and among others on the neutrino signal $[5,11,14,15]$. We do not discuss this point in the present paper since we are mainly interested in a high density and high temperature extension of the EOS.

Up to know, there are less attempts to improve the high density $\left(n_{B} \gtrsim n_{0}\right)$ and high temperature $(T \gtrsim 20$ $\mathrm{MeV}$ ) part of the EOS, although there are many indications that probably the physics of the standard EOS is too poor in this regime, too. First of all, our knowledge about the quantum chromodynamics (QCD) phase diagram suggests a transition to the quark-gluon plasma (QGP) within the range of densities and temperatures reachable in core collapse events, i.e. within the range of our tables. Of course, there are lots of uncertainties about this phase transition, so that its occurrence cannot be affirmed, but the possibility has to be kept in mind when employing a purely nuclear EOS such as the two EOSs by Lattimer and Swesty [3] (LS EOS) or by Shen et al. [4] (Shen EOS) up to densities well above nuclear matter saturation density and temperatures as high as several tens of $\mathrm{MeV}$. There is some first work including this phase transition, see [16]. Secondly, even without thinking about a QCD phase transition, other forms of (non-nucleonic) matter should appear at high densities and temperatures. Already for a long time for cold EOS used for neutron star models, hyperons, pions and kaons have been considered. At temperatures above about 20 $\mathrm{MeV}$, this point becomes even more crucial. This has been confirmed by the first attempts to include hyperons and pions in the Shen EOS [4] for simulations, see [17-19]. The effect of these high density and high-temperature extensions of the EOS on the simulations is not negligible, see e.g. [16, 17, 20, 21]. Let us in particular mention that Sagert et al. [16] found that the QCD phase transition could induce a second shock wave which in their simulations leads to a successful explosion. We shall discuss here the construction of a new EOS including hyperons and pions based on the Lattimer and Swesty [3] (LS) EOS and the effects on some thermodynamic quantities important for the simulations.

The paper is organized as follows. In Section II we briefly recall the basics of the LS EOS [3] upon which our

obtained easily just by multiplying the number density by a constant mass, e.g. the neutron mass $m_{n}$, see the comment on this point in [5], too. model is based. In Section III we present our extension including hyperons and pions. In the following Section, Section IV we discuss the existing constraints we have on the construction of the extended EOS. In particular we discuss the compatibility with the recent observation of a neutron star with almost two solar masses [22], claimed to exclude the existence of additional particles such as hyperons, mesons or quarks within cold neutron stars. Section $\mathrm{V}$ gives an illustration of the usability of the hyperonic EOSs at zero temperature and beta equilibrium, Sec. VI is devoted to a discussion of the results at finite temperature and we conclude in Section VII.

\section{THE LATTIMER AND SWESTY EQUATION OF STATE}

Let us start the description of our model for the extended EOS with a description of the original EOS by [3]. We have chosen this EOS as basis for our work in order to have an approach for the hadronic interaction at hand different from the attempts to include hyperons in the Shen EOS, employing a relativistic mean field model [17-19]. The motivation is of course that there are large uncertainties on the hadronic interaction, so that it is interesting to compare two different types of models. In addition, the LS EOS is one of the two commonly used EOSs in computational astrophysics, so that a comparison of existing results in the literature with results from our extended model should be simplified.

As mentioned above, the LS EOS[3] models the matter as a mixture of one (average) heavy nucleus, $\alpha$ particles, free nucleons, electrons, positrons and photons. Electrons and positrons are treated as non-interacting relativistic gas in pair equilibrium, neglecting electronscreening effects; photons are treated as an ideal ultrarelativistic gas. Equilibrium with respect to strong and electromagnetic interactions is supposed, while no $\beta$ equilibrium is assumed, as expected during core-collapse supernova.

Concerning the nuclear part, the LS EOS follows the works by Lattimer et al. [23] and by Lattimer and Ravenhall [24]. Some simplifications have been made with respect to Refs. [23, 24], e.g., the neutron skin is neglected and a simpler momentum-independent nucleonnucleon interaction is employed instead of a standard non-relativistic Skyrme parameterization. Within the inhomogeneous phase at low density, nuclei are supposed to arrange themselves in a body centered cubic lattice which maximizes the separation of ions. According to the Wigner-Seitz approximation, each ion is at the center of a neutral-charged cell, surrounded by a gas of free nucleons, $\alpha$ and electrons. Interactions between the outside gas and the nuclei are taken into account through an excluded volume. Nucleons are treated as non-relativistic particles; $\alpha$-particles as hard spheres of volume $v_{\alpha}=24 \mathrm{fm}^{3}$ forming an ideal Boltzmann gas. As the density increases, nuclei undergo geometrical shape deformations, 
until they dissolve in favor of homogeneous nuclear matter above approximately saturation density. The formation of non-spherical nuclei ("pasta-phase") is described by modifying the Coulomb and surface energies of nuclei, as discussed in Section 2.8 of Ref. [3]. The phase transition to bulk nuclear matter is treated by a Maxwell construction between the two phases. The configuration of matter and the balance between the different phases is given by the most thermodynamically favorable state, i.e. the one which minimizes the Helmholtz free energy of the system. This procedure, minimizing the free energy, guarantees that the LS EOS is thermodynamically consistent.

Let us stress, however, one point concerning the description of the transition between homogeneous and inhomogeneous matter in the LS EOS. As discussed in Ref. [12], it is not satisfactory, since for all subsaturation densities matter can be viewed as a mixture of nuclei and free nucleons with consequences on the thermodynamic properties. In particular, all thermodynamic quantities are perfectly continuous. As mentioned already in the context of the distribution of nuclei in the inhomogeneous phase, our main interest here is a discussion of the high density and high temperature part, taking a consistent and commonly used EOS for the remaining part.

\section{A. Characteristics of the Lattimer and Swesty equation of state}

The nuclear interaction in the LS EOS contains several parameters, which have been chosen to reproduce reasonable values for properties of symmetric (i.e. equal number of protons and neutrons) bulk nuclear matter at saturation density, for details see the original work [3]. These quantities are related to a power-series expansion of the energy per baryon around saturation density at zero temperature and for symmetric matter:

$$
\begin{aligned}
\frac{E}{A}= & -B+\frac{1}{18} K x^{2}+\frac{1}{162} K^{\prime} x^{3}+\ldots \\
& +\beta^{2}\left(J+\frac{1}{3} L x+\frac{1}{18} K_{\text {sym }} x^{2}+\ldots\right)+\ldots
\end{aligned}
$$

where $x=n_{B} / n_{0}-1$ is the deviation of the baryon number density from saturation and $\beta=\left(n_{n}-n_{p}\right) / n_{B}=$ $1-2 Y_{p}$ describes the asymmetry. The properties of the EOS are thereby given by the values of the coefficients, $n_{0}, B, K, K^{\prime}, J, L$. Of course, this can only give an indication on the general behavior of the EOS, since they are defined at saturation density and for symmetric matter, whereas in the context of neutron stars and core collapse events very asymmetric matter at very different densities is encountered.

Nuclear experiments give constraints on the properties of the saturation density, $n_{0}$, the binding energy, $B$, the incompressibility, $K$ and the symmetry energy at saturation, $J$. Typical values for $n_{0}$ lie in the range $0.15 \mathrm{fm}^{-3}<n_{0}<0.17 \mathrm{fm}^{-3}$ and the binding energy is
15.6 $\mathrm{MeV}<B<16.2 \mathrm{MeV}$. The value of $K$, roughly speaking, determines the stiffness of the EOS, the higher the value of $K$, the stiffer the EOS. But, as mentioned above, it is determined at saturation density and for symmetric matter, such that this interpretation has to be regarded with caution. Nuclear physics experiments on the breathing modes like the isoscalar giant monopole resonance give a value for $K$ at saturation density of $240 \pm 10 \mathrm{MeV}$ [25]. The obvious error is rather small, the result is, however, not uncontested. In particular, the extraction of this value from data on isoscalar giant monopole resonances depends on the density dependence of the nuclear symmetry energy, a quantity under intensive debate in recent years. We thus think that a larger range of values has to be considered. The commonly assumed range for the symmetry energy is $28 \mathrm{MeV}<J<34 \mathrm{MeV}$. For the other parameters, the skewness coefficient $K^{\prime}$, the symmetry energy slope coefficient $L$ and the symmetry incompressibility $K_{\text {sym }}$ data are not really constraining the value.

The original LS EOS [3] uses $n_{0}=0.155 \mathrm{fm}^{-3}, B=$ 16.0 $\mathrm{MeV}$ and $J=28.6 \mathrm{MeV}$, values in reasonable agreement with the constraints ${ }^{2}$. With the original routines, see [26], three sets of boundary and Maxwell construction tables are provided corresponding to three different values of the nuclear incompressibility modulus, $K=180,220,375 \mathrm{MeV}$. Following the above discussion, the two extreme values for $K$ used in the LS EOS are in principle disfavored and the preferred parameter set for simulations should be that with $K=220 \mathrm{MeV}$. We shall, however, keep the two other sets for two reasons. The first one is purely historical: in many simulations the parameter set with $K=180 \mathrm{MeV}$ has been used, so that for comparison with the existing literature it is interesting to have this value at hand. The second one is, as discussed above, that the narrow range, $K=240 \pm 10$ $\mathrm{MeV}$ is not uncontested. In that sense, the range of values of the LS EOS represents an extreme variation of the nuclear parameter sets, i.e. it can give an indication about the uncertainties in the simulations, coming from the uncertainties on the nuclear part of the EOS and we shall in principle keep all three values.

Finally, for simplicity we assume that the nucleon effective mass is equal to the bare mass: $m^{*}=m$. In Lattimer \& Swesty [3], the (density dependent) effective mass term is kept in the equations, so that this assumption can be generalized. Indeed, mean field theories predict an average effective mass $m^{*} / m$ around 0.6-0.8 (see e.g. Bender et al. [27] for a review, and references therein). It has been shown that the inclusion of a temperature dependent nucleon effective mass in nuclei, coming from dynamical correlations beyond mean field, may affect the core-collapse dynamics $[28,29]$. However, as mentioned

\footnotetext{
2 The value of $J$ slightly differs from the one given in Ref. [3] $(J=29.3 \mathrm{MeV})$, see Table 2 in Ref. [5] too.
} 
earlier, the details of the nuclear part are not the aim of the present work, and we shall keep the original version of Lattimer and Swesty [3].

Note, however, one minor correction with respect to the original code. It has been recognized [30, 31] that the original LS EOS underestimated the fraction of $\alpha$ particles. The reason is that the $\alpha$-particle binding energy $B_{\alpha}$ has to be measured with respect to the neutron mass, as all other energies.

Let us now show the results of this correction to the LS routine. In Fig. 1 are displayed the abundances, the entropy and pressure as functions of density, for $K=180 \mathrm{MeV}, Y_{q}=Y_{p}=Y_{e}=0.3$ and for different temperatures $(T=1,2,3 \mathrm{MeV})$. Note that in this case the hadronic charge fraction is given by the proton fraction and that it is equal to the electron fraction, $Y_{e}=\left(n_{e^{-}}-n_{e^{+}}\right) / n_{B}$ due to charge neutrality. These are typical conditions that can be found in a core-collapse supernova simulation. We observe, as expected, that in the original LS routine the abundance of $\alpha$ particles is underestimated; as a consequence, nuclei and free nucleon abundances are higher at a given density. The pressure of the system (upper-left panel) is not very much affected by the corrections to the LS routine, since in this density range the contribution from leptons is the dominant one. Fig. 1 can be directly compared with Fig. 4 of Ref. [30], where the authors plot the results obtained by the original LS EOS and by their 4-species (neutrons, protons, $\alpha$ and ${ }^{54} \mathrm{Mn}$ as representative heavy nucleus) EOS derived assuming nuclear statistical equilibrium (NSE). The results obtained by our tables and by the NSE EOS introduced in Ref. [30] agree. Nevertheless, differences have to be noticed, especially for $T=1 \mathrm{MeV}$; this could be explained by the fact that in their 4-species EOS, the authors assume ${ }^{54} \mathrm{Mn}$ to be the representative heavy nucleus, while in LS EOS the mean nucleus varies as a function of density in order to satisfy the energy minimization condition. This affects the relative abundances and the macroscopic properties of the system.

The lowest values of the density in the original routines for the LS EOS is $n_{B}=10^{-6} \mathrm{fm}^{-3}$. The physical reason is that, in principle at low densities and temperatures below roughly $0.5 \mathrm{MeV}$, an EOS depending only on temperature, baryon number density and charge fraction to describe matter in thermodynamic equilibrium is not sufficient and a nuclear reaction network has to be used. For many purposes, however, a detailed description of matter in this regime is not necessary, and it is thus interesting to have an EOS at hand for this regime. Recently, O'Connor and Ott [32] have generated an EOS table in which they employ the LS EOS for densities above the limiting value of the routines, and, for lower densities, the Timmes EoS [33], under the assumption that matter is composed of an ideal gas of electrons, photons, neutrons, protons, $\alpha$-particles and heavy nuclei with the average $A$ and $Z$ given by the LS EOS at the transition. We shall follow a slightly different approach, see Section III A.

\section{MODEL FOR THE EXTENDED EQUATION OF STATE}

\section{A. Low density regime}

At the densities below the limit of the LS EOS, i.e. $n_{B}=10^{-6} \mathrm{fm}^{-3}$, at low temperatures matter is composed of a gas of nuclei and electrons. At temperatures above roughly $1 \mathrm{MeV}$, nuclei are dissolved in favor of free nucleons, see Fig. 2, where the boundaries between homogeneous and inhomogeneous nuclear matter are shown.

In this regime, the densities are so low, that the different particles are only very weakly interacting and a description in terms of an ideal gas is completely sufficient. This is the reason why the choice of O'Connor and Ott [32] to employ the Timmes EOS in this regime (which is nothing else than an ideal gas of different species) is well justified. We follow the same idea, the only difference is that we make another choice for the matter composition, in particular for the nuclei present and for the matching with the LS EOS at higher densities. O'Connor and Ott take one average nucleus with $A$ and $Z$ obtained from the average heavy nucleus of the LS EOS at the transition density. In this way, it is however not possible to describe a potential variation in $A$ and $Z$ of the mean nucleus throughout the density range covered by this low density extension of the EOS. We therefore use a NSE approach, allowing in principle for many different nuclei to appear. The setup strongly follows the NSE statistical model of Hempel and Schaffner-Bielich [9], with the only exception that we do not consider any excluded volume correction because it is not relevant for such low densities. In particular, we take into account the Coulomb and temperature corrections to the binding energies of the nuclei in order to obtain reasonable transition temperatures to homogeneous matter.

Concerning the matching to the LS EOS at higher densities, we have chosen a matching density of $n_{B}=$ $5 \times 10^{-8} \mathrm{fm}^{-3}$. Although the LS EOS reproduces well the character of matter at this density, i.e. an almost ideal gas of nuclei, photons and electrons, the change of the EOS induces small discontinuities in the thermodynamic quantities, due to the different treatment of the nuclear part. Since the pressure in this regime is dominated in any case by the electron pressure, the discontinuity in the pressure is completely negligible. This is not the case for the energy density and this is the reason why O'Connor and Ott [32] introduce a constant shift in the energy density. We judge that the discontinuity is small enough, such that this shift, problematic in a general relativistic framework, is not necessary.

\section{B. High density regime}

We have added to the LS EOS pions, muons and hyperons. For the first two, no interaction has been assumed and they have just been added as a free gas, satisfying the 


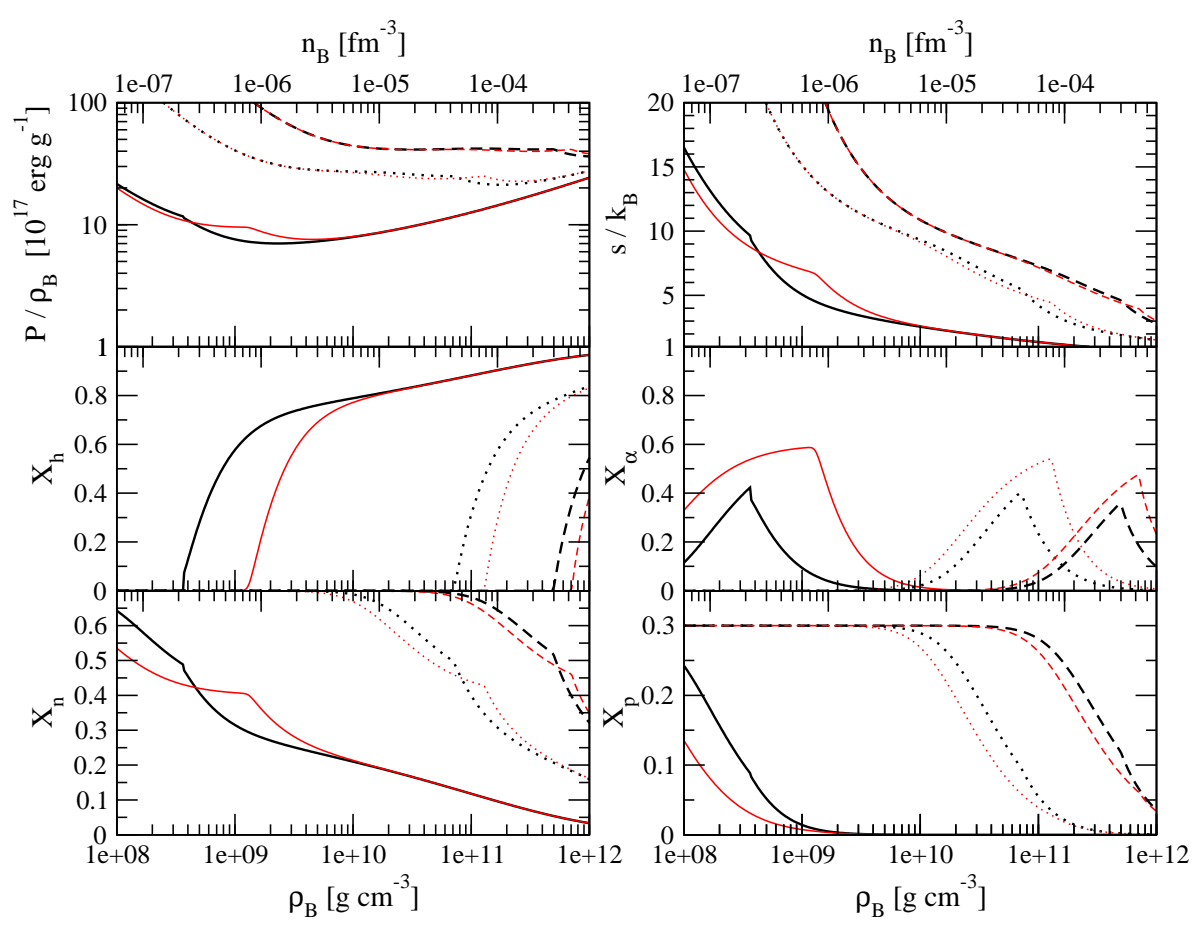

FIG. 1: (Color online) Pressure, entropy and abundances (nuclei, $\alpha$ particles, free neutrons and protons) as a function of density, for $K=180 \mathrm{MeV}, Y_{q}=0.3$ and $T=1 \mathrm{MeV}$ (solid lines), $2 \mathrm{MeV}$ (dotted lines) and $3 \mathrm{MeV}$ (dashed lines). Thick black lines correspond to the results of the original LS routine, while thin red lines correspond to the values obtained with the modified routine. The differences arise from the correction to the binding energy of $\alpha$ particles. We have used here the mass density, defined as $\rho_{B}=m_{u} n_{B}\left(m_{u}\right.$ being the atomic mass unit), for better comparison with the results of Ref. [30].
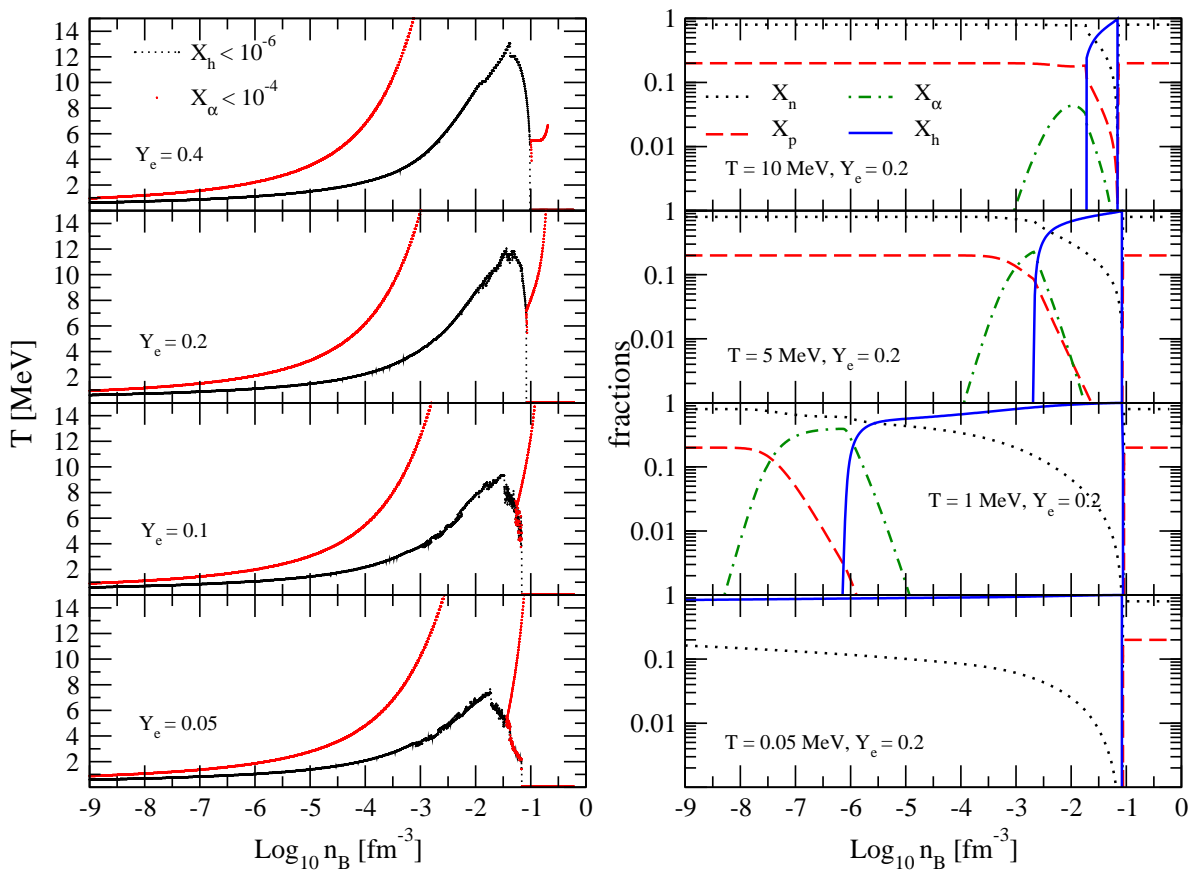

FIG. 2: (Color online) On the left panels, boundaries between homogeneous and inhomogeneous nuclear matter (temperature versus baryon number density) for different $Y_{q}=0.05,0.1,0.2$ and 0.4 are displayed. On the right panels, mass fractions (free neutrons $X_{n}$, free protons $X_{p}$, alpha particles $X_{\alpha}$, and nuclei $X_{h}$ ) as a function of baryon number density are shown for different temperatures $T=0.05,1,5,10 \mathrm{MeV}$, and fixed $Y_{p}=0.2$. 
overall constraint on charge neutrality. There are many works considering these additional particles in cold neutron star cores, see e.g. [34-40]. As already mentioned earlier, there is less work in the context of hot and dense matter in core collapse events, although the possibility of a delayed collapse to a black hole induced by a transition to hyperonic matter has been considered in [41, 42] or pions (and to less extent kaons, too) have already twenty years ago been considered as possible candidates for the hot and dense matter in supernova cores, see e.g. [43]. The authors of Ref. [43] argue that the presence of pions could increase the temperature of the supernova core and increase the number of electron neutrinos and thus lead to a higher neutrino luminosity in favor of a successful explosion. This idea has, however, not been further pursued since there are uncertainties about the employed pionnucleon interaction. It is now commonly assumed, that there is an $s$-wave $\pi N$ repulsion, reducing strongly the number of pions eventually present in supernova cores, thus decreasing the effect described by [43]. This repulsive interaction prevents probably pions from condensing in cold dense matter as discussed for neutron stars, too. It is, however, known that the pion gas is one of the main components for matter in heavy ion collisions. The difference is of course, that for heavy ion collisions much higher temperatures (of the order of $100 \mathrm{MeV}$ or more) are reached and the baryon number densities are much lower than in the core of neutron stars. Core collapse events with massive progenitors are situated somewhat in between, temperatures can reach the order of $100 \mathrm{MeV}$, but densities of several times nuclear matter saturation density are encountered. Here, we mainly discuss the effect of pions on the EOS at high temperature where the pion gas should be a reasonable approximation. Obviously, without interaction, we cannot prevent a $\pi^{-}$ condensate to form below some critical temperature, depending on the density, but we consider introducing a realistic pion-nucleon interaction for cold dense matter to be beyond the purpose of the present paper.

Concerning the muons we have to mention the following point. Since lepton flavor conversion via neutrino oscillations is most probably negligible for core collapse during the first few seconds [44, 45], muon lepton number is conserved independently of electron lepton number. Therefore in principle a muon fraction, $Y_{\mu}=$ $\left(n_{\mu^{-}}-n_{\mu^{+}}\right) / n_{B}$ should be added as variable to the EOS, in addition to the electron fraction $Y_{e}=\left(n_{e^{-}}-n_{e^{+}}\right) / n_{B}$, temperature and baryon number density. This would, however, mean that the simulation codes should evolve muon number, too. For the moment this has not been done for several reasons. One is of course the technical difficulty in adding another evolved quantity together with an additional dimension for the EOS. The second is that one expects the number of muons to be much smaller than the number of electrons due to the mass which is higher by about a factor 200, such that the influence of the muons should not be very important. With these remarks of caution we show in Sec. VI, under the assumption that muons are in thermal equilibrium, some examples where the densities and temperatures are high enough so that the effects of muons are not negligible.

The contribution of pions, muons, electrons and photons to the pressure are obtained from the expression for an ideal gas,

$$
p(\mu, T)=\int \frac{d^{3} p}{(2 \pi)^{3}} \frac{p}{E} \frac{1}{\exp \left[\beta\left(E-\mu_{i}\right)\right] \pm 1},
$$

where $\beta=1 / T$ denotes the inverse temperature, $\mu_{i}$ is the chemical potential for particle $i$ and $E=\sqrt{m_{i}^{2}+\vec{p}^{2}}$ is the single particle energy. The upper sign corresponds to bosons and the lower to fermions.

Hyperons are added by extending the model by Balberg and Gal [46] to finite temperature. This model is a nonrelativistic potential model with the contribution of the interaction between particles of type $i$ and $j$ to the energy density given by:

$$
\begin{aligned}
\varepsilon_{p o t}\left(n_{i}, n_{j}\right)= & \left(1-\frac{\delta_{i j}}{2}\right)\left(a_{i j} n_{i} n_{j}+b_{i j} t_{i} t_{j} n_{i} n_{j}\right. \\
& \left.+c_{i j} \frac{1}{n_{i}+n_{j}}\left(n_{i}^{\gamma_{i j}+1} n_{j}+n_{j}^{\gamma_{i j}+1} n_{i}\right)\right)
\end{aligned}
$$

where $n_{i}$ denotes the baryon number density of species $i$ and the factor $1 / 2$ has been introduced in order to avoid double counting for the interaction between particles of the same type. $t_{i}$ represents the third component of the isospin of the respective particle. $a_{i j}, b_{i j}, c_{i j}$, and $\gamma_{i j}$ are the parameters defining the interaction.

The total baryonic energy density is given by the sum of the potential energy, the mass energy,

$$
\varepsilon_{m a s s}=\sum_{i} n_{i} m_{i},
$$

and the kinetic energy,

$$
\varepsilon_{k i n}=\sum_{i} \frac{\tau_{i}}{2 m_{i}} .
$$

Note that, in order to remain consistent with the LS EOS, we do not take an effective baryon mass into account.

In order to extent the model to finite temperature we did not change the structure of the interaction, but we only replaced the expression for calculating the densities by its finite-temperature version,

$$
n_{i}=\int \frac{d^{3} p}{(2 \pi)^{3}} \frac{1}{\exp \left[\beta\left(E_{i}-\mu_{i}\right)\right]+1} .
$$

$E_{i}=p^{2} /\left(2 m_{i}\right)+U_{i}+m_{i}$ thereby denotes the singleparticle energy for particle $i$. The kinetic energy densities are written in an analogous way,

$$
\tau_{i}=\int \frac{d^{3} p}{(2 \pi)^{3}} \frac{p^{2}}{\exp \left[\beta\left(E_{i}-\mu_{i}\right)\right]+1},
$$

see Eq. (8) in Ref. [46], too. This simple approach of course neglects possible effects of the temperature on the 
(phenomenological) interaction. Investigating these effects is, however, beyond the scope of the present paper.

The single particle potentials $U_{i}$ are obtained from the energy density as $\partial \varepsilon_{\text {pot }} / \partial n_{i}$. The chemical potentials for the different particles are obtained from the following relation

$$
\mu_{i}=B_{i} \mu_{B}+Q_{i} \mu_{q}+L_{i}^{e} \mu_{l_{e}}+L_{i}^{\mu} \mu_{l_{\mu}}+S_{i} \mu_{s},
$$

with $B_{i}, Q_{i}, L_{i}^{e / \mu}, S_{i}$ denoting the baryon number, charge, lepton number and strangeness of particle $i$. Note that we use the relativistic definition of the chemical potentials, i.e. the particle rest mass is included in the chemical potential. This is the reason why we have added the rest mass to the energy $E_{i}$, too. We assume that the reaction processes involving hyperons, e.g. the dominant $\Lambda$ production reaction, $n+n \rightarrow n+K+\Lambda$ are in equilibrium. In addition, we assume equilibrium for strangeness changing (weak) interactions, such that we can take the strangeness chemical potential $\mu_{s}=0$.

We have slightly modified the values of parameters for the hyperon-nucleon (YN) and hyperon-hyperon (YY) interaction with respect to the work by Balberg and Gal [46] in order to be compatible with current experimental data on hypernuclei, see Section IV A. This model has the great advantage that hyperons are added on a nuclear interaction which is exactly the same as in the original LS EOS by [3], so that an "artificial phase transition", induced only by matching one nuclear model to another and which is thus completely unphysical, is avoided.

\section{CHOICE OF THE PARAMETERS}

This section will be devoted to a discussion of the existing constraints on the choice of the parameters for the extended EOS. We start with hyperonic data and then have a look on cold neutron stars.

\section{A. Hyperonic data}

In contrast to the nuclear data, hyperonic data are extremely scarce, so that there are large uncertainties on the hyperonic interactions. Starting with the description of the fundamental hyperon-nucleon $(Y N)$ interaction, it is a long way off having reached the same precision as the nucleon-nucleon $(N N)$ interaction, mainly because of the very limited amount of scattering data. On the theoretical side, chiral effective field theory calculations have been performed, too [47, 48], improving on the reliability of the $Y N$ potentials, but still being far from giving conclusive results. First results from lattice QCD simulations of the $Y N$ interaction have become available [49], too.

Concerning the properties of hyperons in dense nuclear matter, there are on the one hand many-body calculations, starting from the fundamental interaction. In addition to the traditional $G$-matrix calculations [50-52], recently a Hartree-Fock calculation based on a $V_{\text {lowk }}$ potential has been presented in Ref. [53]. The results are in reasonable agreement between the different approaches, but large uncertainties remain due to the not very well known fundamental interaction, see e.g. the discussion in Ref. [53]. It has been shown, too, that the inclusion of hyperonic three-body forces $(Y N N)$ do not strongly change the results [54]. We should, however, mention that there are very large uncertainties on the hyperonic three-body force. The authors of Ref. [54] assume an effective phenomenological form similar to the interaction in the model by Balberg and Gal [46] we are employing here. They, however, limit the strength of the three-body force arguing that it should be less important than the nuclear one. Here we follow a slightly different philosophy: we think that the hyperonic three-body force is not known well enough to put any constraint on it ad hoc. We shall thus limit it only by data and by neutron star observations, see next section.

On the other hand, single-particle potentials $V_{Y N}$ in symmetric nuclear matter have been extracted from data on hypernuclei. In that way the empirical value for the $\Lambda N$-potential at saturation density $\left(n_{B}=n_{0}\right), V_{\Lambda N} \approx$ $-30 \mathrm{MeV}$, has been obtained. This value is in agreement with an analysis of $\left(\pi^{-}, K^{+}\right)$inclusive spectra on different target nuclei [55] and reproduced by most many-body calculations, so that it is commonly accepted. Balberg and Gal [46] have adjusted their parameters to this value, too. For $\Sigma^{-}$, the situation is somewhat ambiguous. The observation of a ${ }_{\Sigma}^{4} \mathrm{He}$ bound state [56] requires an attractive potential, whereas the analysis of the $\left(\pi^{-}, K^{+}\right)$ inclusive spectra $[55,57]$ indicate a repulsive potential, possibly up to a value of $V_{\Sigma N}=100 \mathrm{MeV}$ at saturation density. Theoretical many-body calculations show a large variety of results, too, see e.g. [53], ranging from slightly less attractive values as the $\Lambda N$ case to strongly repulsive values of up to several tens of $\mathrm{MeV}$. Balberg and Gal [46] adopt two different versions, one with an attractive potential of the same form as for the $\Lambda$, and another local potential form giving rise to a repulsive potential. We here take the form given in Eq. (3), but choosing the parameters in order to obtain a repulsive single-particle $\Sigma N$ potential. Concerning the $\Xi N$ singleparticle potential, less data are available. Only a few events of $\Xi$-hypernuclei have been observed, so that it is much more difficult to reliably fix the well depth of the single-particle potential. Balberg and Gal [46] take a range $V_{\Xi N}=(-20)-(-25) \mathrm{MeV}$, whereas newer data indicate a less attractive potential of $V_{\Xi N} \approx-14 \mathrm{MeV}$ [58].

Concerning the hyperon-hyperon $Y Y$ interaction, the situation is rather difficult. Early experiments interpreted in terms of production of several double $\Lambda$ hypernuclei indicate a rather strong attractive potential of the order of $V_{\Lambda \Lambda} \approx-40 \mathrm{MeV}$ [46]. More recent measurements [59] are in favor of much lower values, $V_{\Lambda \Lambda} \approx-10 \mathrm{MeV}$. For other hyperons, no data are available. We shall take, in view of the faint knowledge, either no $Y Y$-interaction at all or a universal $Y Y$ interaction 
with different values for the well depth. For the isospin dependent terms we follow Balberg and Gal [46].

\section{B. Neutron stars}

In the center of neutron stars densities of several times nuclear matter saturation density are reached, so that they present an important test for the EOS of matter above $n_{0}$. In contrast to the hot core-collapse environment, neutron stars older than several minutes can be regarded as cold from the EOS point of view since the temperature reached is well below $1 \mathrm{MeV}$. In addition, $\beta$-equilibrium is achieved and neutrinos can freely leave the system, so that the EOS in this case is only a function of baryon number density.

In particular observed masses put constraints on the EOS. There are a number of precise mass measurements from neutron stars in binary systems, for a compilation see e.g. Ref. [1]. For a given EOS and a given central density, the mass and radius of a non-rotating neutron star can be obtained by solving the equations for hydrostatical equilibrium together with Einstein's equations. In this case of a spherical star it just gives the TOVsystem. It is known for a long time, that theoretical many-body EOS with hyperons predict maximum masses of the order $1.4 M_{\odot}$ or below, incompatible with many precisely known neutron star masses [60-62]. The recent precise measurement of PSR 1614-2230 with a mass of $1.97 \pm 0.04 M_{\odot}$ [22] thus completely excludes those EOSs. The authors of Ref. [22] claim that their data excludes any type of EOS with "exotic" contributions, i.e. other particles than nucleons and electrons. The argument is obvious and well known: adding new degrees of freedom to the EOS softens it and thus the maximum mass decreases. This simple argument is, however, only true without interaction. As has already been shown for EOS with a transition to quark matter [63, 64], a repulsive interaction can cure the problem and allow for neutron star maximum masses of $2 M_{\odot}$ or even above. For hyperons, this seems more difficult, and within the microscopic approaches, the origin of the necessary repulsion at high densities has not yet been found. Recently different RMF models have been presented which successfully reconcile hyperonic matter with PSR 16142230, see [34, 35, 65-67].

Here, we take a phenomenological point of view and we choose parameters for the model by Balberg and Gal [46] compatible on the one hand with hyperonic data and on the other hand with PSR 1614-2230, without looking for a deeper understanding of the repulsion. Thus, we shall show that it is possible to reconcile the data, in particular a neutron star mass of $2 M_{\odot}$ with the existence of hyperons in dense matter, but without answering the question why theoretical many-body calculations, predicting the existence of hyperons at densities of about $2-3 n_{0}$, cannot reproduce the $2 M_{\odot}$ neutron star. The values of the different single-particle potentials and the maximum mass

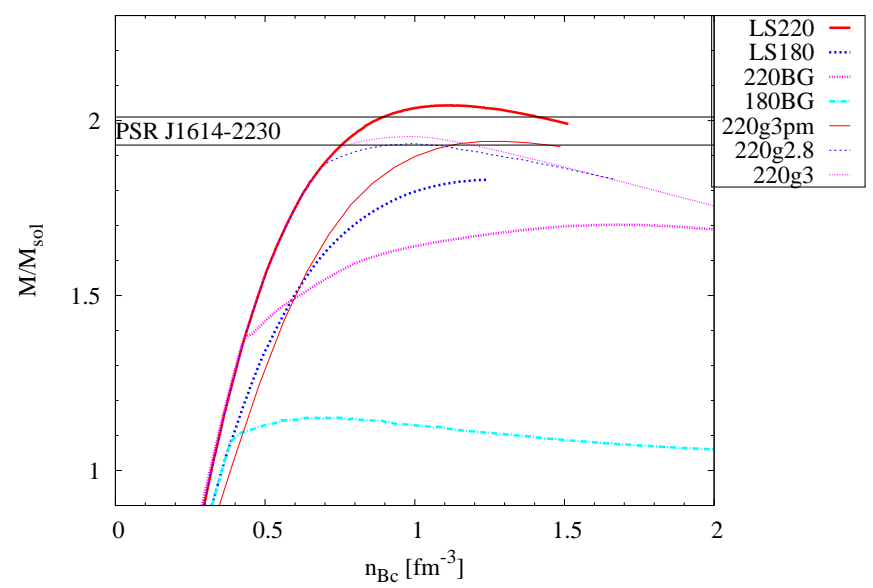

FIG. 3: (Color online) Gravitational mass of non-rotating spherical neutron stars as a function of central baryon number density for the different equations of state discussed in the text.

of a spherical neutron star for different parameter sets are listed in Table I. The first three digits in the name of the parameter set thereby indicate the value of the incompressibility modulus of nuclear matter. In Table II we list the corresponding parameters for the hyperonic interaction. The neutron star masses as a function of central density are displayed in Fig. 3 together with the constraint from PSR 1614-2230 and the curves obtained with the LS EOS for $K=180$ and $K=220 \mathrm{MeV}$. Note that pions and muons have only been included for $220 \mathrm{pm}$.

Parameter set 180BG takes the softest version of the original Balberg and Gal paper [46] with two exceptions: we modified the $\Sigma$ parameters in order to obtain a slightly repulsive $V_{\Sigma N}$ and we neglected any $Y Y$ interaction. This parameter set of course gives a far too low neutron star maximum mass, we include it, however, for comparison. It can be seen as an extreme case, representing a very (too) soft EOS. Set 220BG takes the version with the strongest high density repulsion for $Y N$ and $Y Y$ parameters from [46], with, again, one exception: $V_{\Sigma N}$ is chosen repulsive. The other three sets show three examples of parameters compatible with PSR 1614-2230, still giving reasonable values for the well depths at saturation density. Let us, however, remark that we did not find any viable parameter set with an attractive potential for $V_{\Sigma N}$. For $220 \mathrm{~g} 2.8$ and $220 \mathrm{~g} 3, V_{\Sigma N}$ is even strongly repulsive, at the limit of what is compatible with the estimates discussed in Sec. IV A. For 220pm it is weaker, but in that case, no hyperons are present in cold neutron star matter. The deviation from the LS EOS case with $K=220 \mathrm{MeV}$, visible in Fig. 3, thereby arises only from the presence of pions and muons. 


\begin{tabular}{|l|c|c|c|c|c|c|} 
Name & $K[\mathrm{MeV}]$ & $M_{\max } / M_{\odot}$ & $V_{\Lambda N}[\mathrm{MeV}]$ & $V_{\Sigma N}[\mathrm{MeV}]$ & $V_{\Xi N}[\mathrm{MeV}]$ & $V_{Y Y}[\mathrm{MeV}]$ \\
\hline \hline 180BG & 180 & 1.15 & -28.2 & 16.8 & -24.3 & 0.0 \\
\hline 220BG & 220 & 1.70 & -26.6 & 28.5 & -22.8 & -38.0 \\
\hline 220g2.8 & 220 & 1.93 & -29.6 & 65.7 & -23.0 & -55.1 \\
\hline $220 \mathrm{~g} 3$ & 220 & 1.95 & -26.8 & 73.0 & -15.3 & -10.3 \\
\hline $220 \mathrm{pm}$ & 220 & 1.94 & -26.8 & 24.1 & -24.5 & -10.3 \\
\hline
\end{tabular}

TABLE I: Properties of the different equations of state discussed in the text. The cold neutron star maximum mass is given for a non-rotating spherical star.

\begin{tabular}{|c|c|c|c|c|c|c|c|c|c|c|c|}
\hline Name & $\begin{array}{c}a_{\Lambda N} \\
\mathrm{MeV} \mathrm{fm}{ }^{3}\end{array}$ & $\begin{array}{c}c_{\Lambda N} \\
\mathrm{MeV} \mathrm{fm}^{3 \gamma}\end{array}$ & 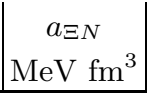 & $\begin{array}{c}c_{\Xi N} \\
\mathrm{MeV} \mathrm{fm}^{3 \gamma}\end{array}$ & $\begin{array}{c}a_{\Sigma N} \\
\mathrm{MeV} \mathrm{fm} \\
\end{array}$ & $\begin{array}{c}c_{\Sigma N} \\
\mathrm{MeV} \mathrm{fm}^{3 \gamma}\end{array}$ & $\begin{array}{c}b_{\Sigma N} \\
\mathrm{MeV} \mathrm{fm}^{3}\end{array}$ & $\begin{array}{c}a_{Y Y} \\
\mathrm{MeV} \mathrm{fm}^{3}\end{array}$ & $\begin{array}{c}c_{Y Y} \\
\mathrm{MeV} \mathrm{fm}^{3 \gamma}\end{array}$ & $\begin{array}{c}b_{\Sigma \Sigma} \\
\mathrm{MeV} \mathrm{fm}^{3}\end{array}$ & $\gamma$ \\
\hline $180 \mathrm{BG}$ & -505.2 & 605 & -434.4 & 520.1 & 10 & 175 & 214.2 & 0 & 0 & 0 & $4 / 3$ \\
\hline $220 \mathrm{BG}$ & -340 . & 1087.5 & -291.5 & 932.5 & 130. & 300 & 214.2 & -486.2 & 1553.6 & 430 & 2 \\
\hline $220 \mathrm{~g} 2.8$ & -270 & 2300 & -170 & 2000 & 500 & 200 & 214.2 & -400 & 1500 & 430 & 2.8 \\
\hline $220 \mathrm{~g} 3$ & -270 & 4000 & -170 & 2900 & 450 & 250 & 214.2 & -90 & 1000 & 430 & 3 \\
\hline $220 \mathrm{pm}$ & -270 & 4000 & -240 & 3400 & 130 & 800 & 214.2 & -90 & 1000 & 430 & 3 \\
\hline
\end{tabular}

TABLE II: Parameter values of the different equations of state discussed in the text.

\section{COLLAPSE OF A COLD NEUTRON STAR TO A BLACK HOLE}

The goal of this Section is to show the numerical usability of the EOS, by implementing cold EOSs presented in Sec. IV B into a numerical code and studying the collapse of a cold neutron star to a black hole. It is not fully relevant to try and measure the effects of the presence of hyperons or additional particles in this case because it is difficult to find comparable physical settings: from Fig. 3 it is clear that the maximal masses with or without hyperons are quite different. Therefore, initial (unstable) neutron star models used as initial conditions are too different with different EOSs to provide comparable collapses to a black hole. The comparison can be performed in the case of a stellar core-collapse, starting from the same main-sequence massive star initial data and following then the collapse, bounce, stalling of the shock and collapse of the proto-neutron star to a black hole, with different EOSs at finite temperature. As this is beyond the scope of the current paper, it shall be the subject of a forthcoming study.

\section{A. Transition to a quark phase}

The physical model that we study here is the collapse to a black hole of an unstable neutron star, i.e. with a central density higher than that corresponding to the maximal mass. During the collapse, the densities reached inside the neutron star can be much higher than several times the saturation density (see Fig. 4 and [68]). As the LS model for the EOS is non-relativistic, it can in principle allow for a sound speed greater than the speed of light. With the numerical code we are using (see Sec. V B hereafter), such a situation can lead to spurious oscillations and instabilities destabilizing the whole simulation. This happens in the LS EOS or in most of the extended EOSs for high densities, typically beyond $5-10 \mathrm{fm}^{-3}$, that is at more than twenty times nuclear matter saturation density. At these high densities, the nature of matter is anyway far from being well known. It seems rather natural to assume that there is a transition to a quark matter phase at some density and this is what we do here. Therefore we have used in our EOS a transition to a simple model for quark matter, the MIT bag model [69] with massless quarks, implying a supraluminal sound speed. The transition is constructed using a Maxwell construction. Within this very simple model the density for the transition can be adjusted by tuning the value of the bag constant. Since the aim of our paper is not to investigate the transition to quark matter (see e.g. [16] for such a study in the context of core collapse), we try to push the transition density as far as possible without having superluminal sound speeds. This means that we choose values of the bag constant much above commonly used values, leading to appearance of quark matter at a density of about $n_{B}=2 \mathrm{fm}^{-3}$, the exact density thereby depends on the specific parametrization of the EOS used.

With the quark phase transition presented here for the zero-temperature EOS, the sound speed always remains lower than the speed of light, suppressing all the possible instabilities coming from superluminal characteristics. In principle this problem could have been avoided by other prescriptions. For example, a causal EOS, i.e. a maximally stiff EOS has been used in this context, too. We, however, think that a transition to quark matter better reflects the physics, although it should be kept in mind 
that in this high density region we do not know much about the real physics and that our quark model is a very simplistic one.

\section{B. Model setup}

We use a general-relativistic hydrodynamics code CoCoNuT [70], solving the continuity equation and the relativistic Euler equations for a perfect fluid by means of high-resolution shock-capturing methods [71]. Einstein equations, in isotropic gauge and maximal slicing are solved on a different grid with spectral methods [72]. This code, although able to perform 3-dimensional simulations is run in spherical symmetry since we are only interested here in illustrating that the different EOSs can be successfully used in simulations of a collapse to a black hole. The EOS is tabulated, read from a file and interpolated for every grid point. Static, spherically symmetric, neutron star initial data are obtained with the same EOS as the one used for the evolution, and with the same gauge, using the non-rotating version of the code described in [73] and the LORENE library [74]. The initial star is chosen to be a spherical configuration on the unstable branch, with a gravitational mass decreasing if one increases central density. An unstable neutron star is not bound to collapse to a black hole: it can expand in order to "migrate" to the stable branch, i.e reach a lower density with the same baryon mass, such that it is stable with respect to radial oscillations (see also [75]).

The star is then perturbed by amplifying the radial density profile by one percent. This procedure ensures that the star will collapse to a black hole and not migrate to the stable branch. The standard picture of such a numerical model is that the star collapses until generalrelativistic effects become dominant (see e.g. [68] for a complete description). Among these is the "frozen-star" effect which comes from our choice of time gauge (maximal slicing), avoiding the appearance of a central singularity. It implies that several quantities do no longer evolve near the center of the star, whereas some of the metric coefficients develop huge gradients, limiting the simulation in time. One sign that evolution is frozen near the center of the star is given by the fact that one metric coefficient, the lapse $\alpha$, representing the ratio between the physical time measured by the Eulerian observer and the coordinate time, is tending toward zero. The second general relativistic effect is the formation of an apparent horizon at a finite distance, growing further outward until including all the neutron star matter. This is an evidence of the formation of a black hole. The 2-surface defining the horizon is tracked in our code by an apparent horizon finder [76], which enables us to compute the baryon mass inside the black hole, too.
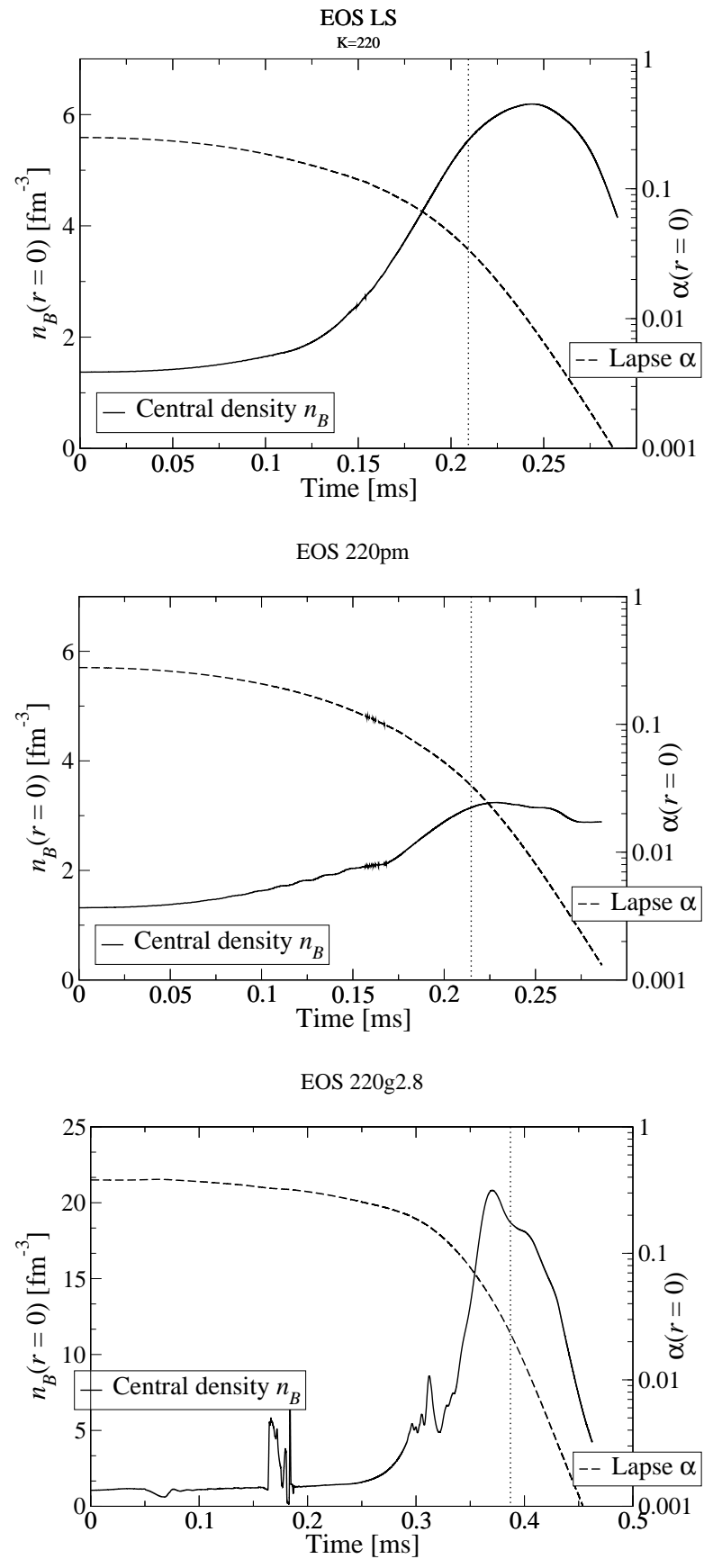

FIG. 4: Profiles of central density (solid line) and central lapse (dashed line) as functions of time during the collapse of a perturbed unstable neutron star to a black hole. Top figure was obtained with the LS EOS [3] with $K=220 \mathrm{MeV}$, the two others by EOSs described in Tabs. I and II. Vertical dotted lines give the time of the formation of the apparent horizon.

\section{Results of simulations}

We have run our code on six different tabulated EOSs displayed in the left column of Tab. III. In this table, LS180 and LS220 stand for the Lattimer-Swesty EOS 


\begin{tabular}{|r|c|c|c|c|c|} 
EOS & $t_{\mathrm{AH}}[m s]$ & $t_{99}[m s]$ & $n_{B}^{\max }\left[\mathrm{fm}^{-3}\right]$ & $\Delta M_{B}$ & $\Delta M_{g}$ \\
\hline \hline LS180 & 0.223 & 0.23 & 18.6 & $2 \times 10^{-6}$ & $4 \times 10^{-3}$ \\
\hline 180BG & 0.188 & 0.216 & 114 & $7 \times 10^{-6}$ & $2 \times 10^{-2}$ \\
\hline LS220 & 0.209 & 0.216 & 6.19 & $2 \times 10^{-6}$ & $4 \times 10^{-3}$ \\
\hline $220 \mathrm{~g} 2.8$ & 0.387 & 0.396 & 20.8 & $5 \times 10^{-6}$ & $6 \times 10^{-3}$ \\
\hline $220 \mathrm{~g} 3$ & 0.327 & 0.334 & 9.51 & $6 \times 10^{-6}$ & $5 \times 10^{-3}$ \\
\hline $220 \mathrm{pm}$ & 0.215 & 0.22 & 3.24 & $10^{-6}$ & $6 \times 10^{-3}$ \\
\hline
\end{tabular}

TABLE III: Characteristics of the six collapses to a black hole studied here. EOS names are detailed in the text, $t_{\mathrm{AH}}$ is the apparent horizon formation time (since the starting of the collapse), $t_{99}$ the time at which $99 \%$ of the baryon mass has gone into the black hole. $n_{B}^{\max }$ is the maximum central density reached during the collapse, $\Delta M_{B}$ and $\Delta M_{g}$ are the relative conservations of baryon and gravitational masses, respectively.

with the incompressibility $K=180$ and $K=220 \mathrm{MeV}$, respectively. The properties of the four other EOSs are given in Tabs. I and II. The EOS labeled 220BG in these tables could not give any reliable result in the simulations, because too much numerical noise appeared already in the initial data. Neutron star collapses with any of the six EOSs listed in Tab. III would lead to the formation of a black hole in a time of a few tenth of a millisecond. Time evolution profiles for the density and the central value of the lapse $\alpha$ are given in Fig. 4 for EOSs LS220, 220pm and 220g2.8. On each of these plots the central density is increasing, eventually with some oscillations coming from the focusing of the initial perturbation, before reaching a maximum value and then decreasing. This decrease is mostly due to the finite resolution at the star center, as the density should tend toward a given value (frozen-star picture). This spurious decrease is not an issue because it appears after (or about the same moment as) the formation of the apparent horizon and therefore in a region inside the black hole that cannot influence the matter still falling onto it. This problem can in principle be cured using the excision technique (removing a neighborhood of the center, replacing it by boundary conditions [77]), which we plan to implement in our code in the near future.

On Fig. 4, the lapse is decreasing toward zero, as expected, and the apparent horizon forms sufficiently soon to let most of matter remaining outside the black hole to enter it. The simulations are ended when the radial gradients of the gravitational fields become too high to be well described with spectral methods. As seen from the values of $t_{99}$ Tab. III, this happens after all matter has been swallowed by the apparent horizon and therefore one is left with a static Schwarzschild black hole, with no evolution outside the horizon. From Fig. 4 and Tab. III, one can notice that the maximal density reached during the collapse strongly depends on the EOS. In particular, the EOSs with an incompressibility modulus $K=180$ $\mathrm{MeV}$ being softer, matter is more compressed. The ad- dition of hyperons makes the EOS even softer and, at the end the collapse with EOS $180 \mathrm{BG}$, it reaches such high densities that they no longer seem realistic (beyond $\left.100 \mathrm{fm}^{-3}\right)$. Finally, Tab. III also gives some error indicators: $\Delta M_{B}$ is the relative variation of the baryon mass (number of particles times their rest mass), $\Delta M_{g}$ is the variation of the system's gravitational mass (as deduced from the asymptotic behavior of the gravitational field). The conservation of baryon mass is directly imposed by solving the equation for the conservation of the baryon current, whereas the conservation of gravitational mass is only an indirect consequence of the solved equations and of the spherical symmetry (no gravitational waves). Therefore, this last indicator is a good estimate of the overall accuracy of a run. From all these results, we can claim that, apart from EOS 220BG and 180BG, the cold EOSs derived here are suitable for numerical simulations in the demanding model of the collapse to a black hole.

\section{RESULTS AT FINITE TEMPERATURE}

Let us now discuss the behavior of the EOS with the different parametrizations at finite temperature. As mentioned earlier, simulations of core collapse with massive progenitors show that rather high temperatures of several tens of $\mathrm{MeV}$ and even more at proto-neutron star densities are reached, see e.g. $[17,78]$. It is clear that the thermal energy is in favor of the production of additional particles such as hyperons and mesons or nuclear resonances. The abundances of hyperons, pions and kaons measured from heavy ion collisions indeed indicate that they are produced during the collisions. One should of course insist on the fact that the conditions in heavy ion collisions are different from core collapse events: the baryon densities are lower and the temperatures are probably slightly higher, the timescales are such that no weak equilibrium for strangeness is achieved and the difference in neutron and proton densities is much less pronounced than in core collapse events or in neutron stars. But the results show clearly that the thermal production of those additional particles is important.

At finite temperature and without assuming $\beta$ equilibrium, the EOS is a function of three variables which are generally chosen to be $T, n_{B}, Y_{e}$. We now discuss the properties of the EOS as a function of these variables. Of course we cannot cover the whole range, but we shall choose some particular conditions. In Fig. 5 we show the different hyperon fractions as functions of electron fraction for a temperature of $T=60 \mathrm{MeV}$ and two different densities, $n_{B}=0.15 \mathrm{fm}^{-3}$ and $n_{B}=0.3 \mathrm{fm}^{-3}$. In the simulations of Sumiyoshi et al. [17] the first density corresponds to the conditions of a collapse with a $40 M_{\odot}$ progenitor at bounce at about $10 \mathrm{~km}$ from the center. Of course the exact thermodynamic conditions in a simulation depend on the EOS, so that this is just to say this is a typical situation within a proto-neutron star after bounce. High densities are typically reached 

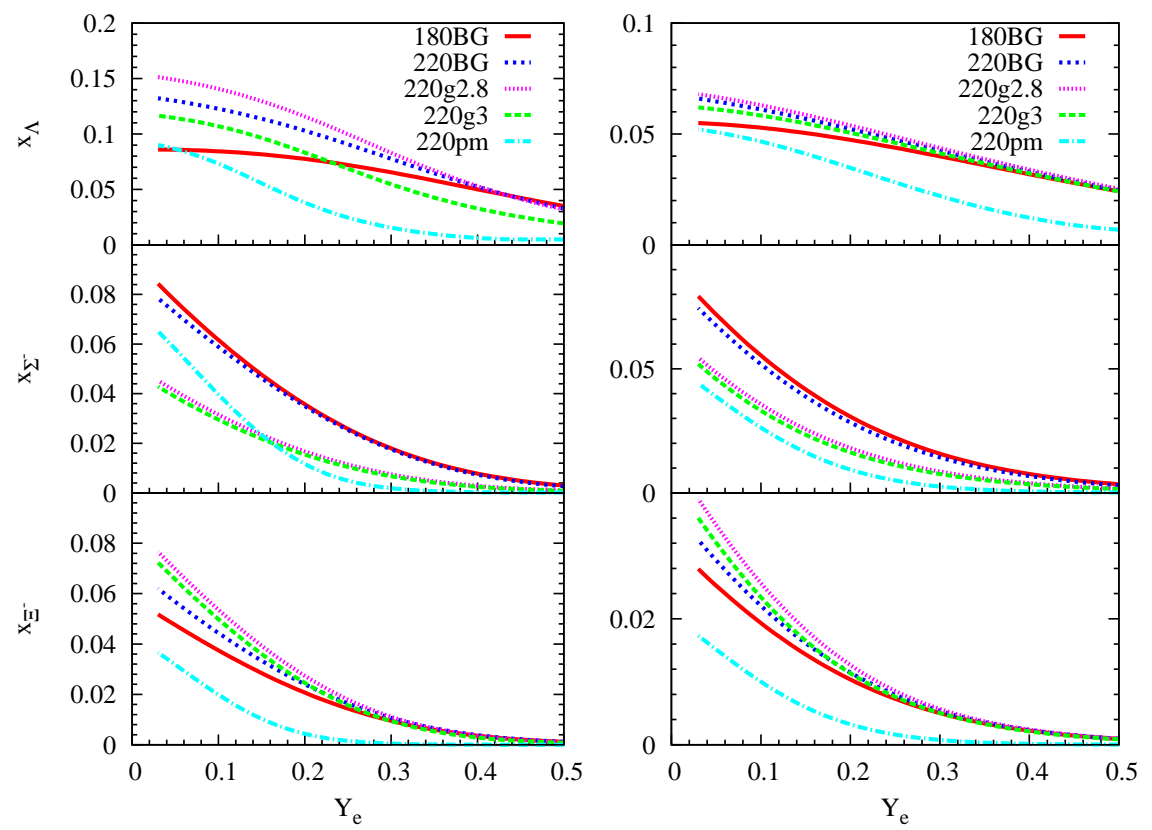

FIG. 5: (Color online) Fractions of different hyperons as a function of the electron fraction at a temperature of $60 \mathrm{MeV}$ and for $n_{B}=0.15 \mathrm{fm}^{-3}$ (right) and $n_{B}=0.3 \mathrm{fm}^{-3}$ (left) corresponding roughly to once and twice nuclear matter saturation density. The fractions of $\Sigma^{0,+}, \Xi^{0}$ are not shown since they are below one percent.

in the post-bounce phase. We only show the fractions of $\Lambda, \Sigma^{-}$and $\Xi^{-}$-hyperons since the fractions of the other hyperons are very small, between one permille and one percent.

At $n_{B}=0.15 \mathrm{fm}^{-3}$, the $\Lambda$ and $\Xi^{-}$fractions show clearly two groups: the parametrizations without pions and muons and the one including them. The reason for the different behavior of the EOS if pions and muons are included is mainly due to the presence of muons. This can be understood rather easily. Under the present conditions muons are almost equally abundant as electrons, so that in order to conserve global charge neutrality, the hadronic charge fraction for a given $Y_{e}$ is increased by almost a factor of two with respect to matter without muons. This is clearly reflected in the curves. Let us stress, however, that the muon fraction is determined by the degeneracy factor $\mu_{\mu} / T$ so that it depends on the ratio of chemical potential and temperature. The curves are calculated assuming the same chemical potential for electrons and muons. In the case that neutrinos can freely leave the system, we have $\mu_{\mu^{-}}=\mu_{e^{-}}=\mu_{q}$, but in the hot proto-neutron star neutrinos are trapped so that there are nonzero lepton number chemical potentials which are not necessarily the same for electrons and muons as mentioned already earlier. In general electron neutrinos are the most abundant ones, so that assuming an equilibrium Fermi-Dirac distribution the lepton number chemical potential for electrons is expected to be higher than that of the other leptons. We therefore expect that in a realistic simulation the chemical potential for muons is lower than that for electrons reducing the number of muons. It is, however, difficult to esti- mate quantitatively the reduction. We can only say that we think that our results represent an upper limit for the importance of the effects muons can have on the EOS.

The $\Sigma^{-}$fraction shows a stronger dependence on the hyperonic interaction. Remind that the two parametrizations $220 \mathrm{~g} 3$ and $220 \mathrm{~g} 2.8$ contain a rather strong repulsion for the $\Sigma^{-}$single-particle potential, see Tab. I, whereas the repulsion is much less strong for the others. This observation explains the reduction of the $\Sigma^{-}$-fraction in these two models with respect to the others. The overall increase in the $\Sigma^{-}$-fraction with decreasing $Y_{e}$ is the well known effect that in neutron rich nuclear matter negatively charged hyperons are favored. To a smaller extent the same is observed for the $\Xi^{-}$, which is, however, less abundant overall due to its higher mass. Altogether the hyperon fractions reach maximum values of about $2-8 \%$.

In Fig. 6 the pressure, the sound speed and the internal energy per baryon with respect to the proton mass $m_{p}$ are displayed for the same densities and temperature. The latter quantity is defined as

$$
\epsilon=\frac{\varepsilon}{n_{B} m_{p}}-1,
$$

with $\varepsilon$ denoting the total energy density. These three quantities are key ingredients for the hydrodynamic simulations. We show the results for the different parametrizations of the hyperonic interactions as well as, for comparison, the LS EOS with $\mathrm{K}=180 \mathrm{MeV}$ and $\mathrm{K}$ $=220 \mathrm{MeV}$, too.

We again observe that the EOS including muons behaves differently from all the others. The reason has been explained above. In particular, the usual softening of the 

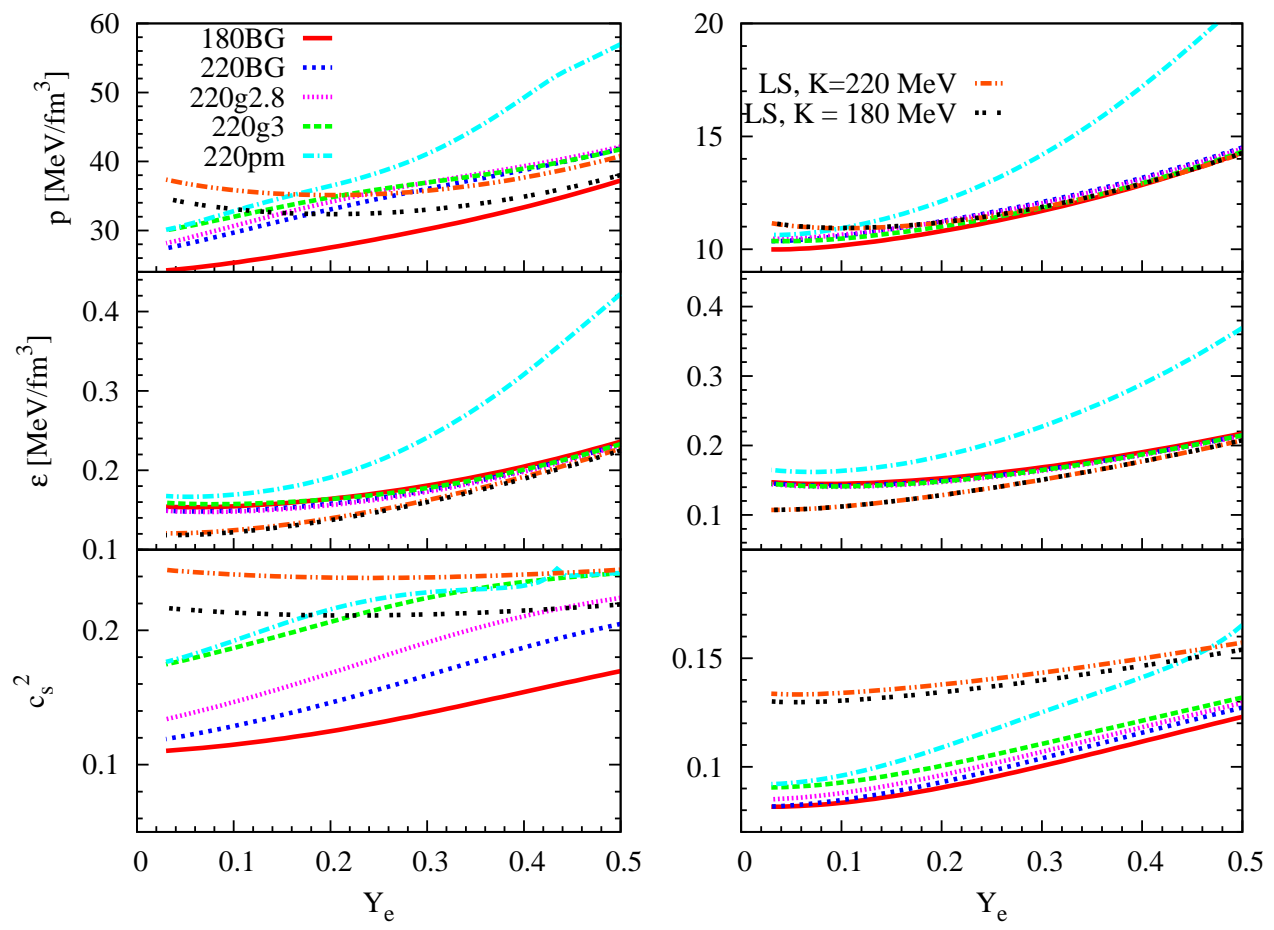

FIG. 6: (Color online) Thermodynamic quantities as a function of the electron fraction at a temperature of $60 \mathrm{MeV}$ and for $n_{B}=0.15 \mathrm{fm}^{-3}$ (right) and $n_{B}=0.3 \mathrm{fm}^{-3}$ (left) corresponding roughly to once and twice nuclear matter saturation density. The upper panels show the pressure, the middle ones the internal energy per baryon with respect to the proton mass (see text for the definition) and the lower ones the sound speed squared.

EOS from additional degrees of freedom by adding different types of particles is not seen. The point is that this softening is overcompensated by the effect of the increased hadronic charge fraction induced by the presence of muons, see above. For the other EOS including only hyperons the softening is indeed seen, as expected it is more pronounced at higher density (left panels). Compared with the purely nuclear EOS $\epsilon$ is higher including hyperons. This is not very surprising neither since the hyperons are more massive than nucleons and therefore replacing a nucleonic state with a hyperonic one in general increases the energy density. The modifications of pressure and energy density due to the presence of additional particles is reflected in the sound speeds, too. As already mentioned above, the hyperon fractions increase with decreasing electron fraction, so that the effects on the thermodynamic quantities increase with decreasing $Y_{e}$, too.

At $n_{B}=0.15 \mathrm{fm}^{-3}$ (right panels) the interaction has only little effect on the thermodynamic quantities, three groups of EOS can clearly be distinguished: the two LS ones, those with hyperons and the EOS including in addition pions and muons. The differences arising from the different interaction between the two LS ones, and between the four hyperonic ones are only very small. This can be understood since at high temperature and low density the kinetic energy should dominate and the interaction terms should in turn be less impor- tant. At higher densities, as can be seen from the figures at $n_{B}=0.3 \mathrm{fm}^{-3}$ (left panels), indeed the influence of the interaction is stronger. The difference in pressure between the two LS ones is of the order of $10 \%$ and between the four hyperonic ones of the order of $15 \%$. It should be stressed, however, that the softest one, 180BG, gives a maximum mass for a cold spherical neutron star below $1.2 M_{\odot}$ and that it is thus not very realistic, see Sec. IV B. The difference between the three remaining hyperonic EOS is much smaller.

At which temperatures the additional particles in the EOS start to play a role? In order to answer this question we display in Fig. 7 the fractions of $\Lambda, \Sigma^{-}$, and $\Xi^{-}$and in Fig. 8 the pressure, $\epsilon$ and the sound speed as a function of temperature. The densities are the same as before, $n_{B}=0.15 \mathrm{fm}^{-3}$ on the right and $n_{B}=0.3 \mathrm{fm}^{-3}$ on the left. We have chosen a relatively low electron fraction, $Y_{e}=0.1$, because we want to show an upper limit case, i.e. an optimistic estimation of the effect of the additional particles on the EOS. At the smaller density hyperons appear at about $25 \mathrm{MeV}$, independently of the EOS used. The first one to appear is the $\Lambda$-hyperon. As expected the hyperon fractions rise with temperature, reaching about $10 \%$ for $\Lambda$ and $\Sigma^{-}$and $5 \%$ for $\Xi^{-}$at a temperature of 100 $\mathrm{MeV}$. The differences in the parametrization of the hyperonic interaction does not induce large differences. Let us make, however, two remarks. First, the particular features of the EOS with pions and muons have already been 

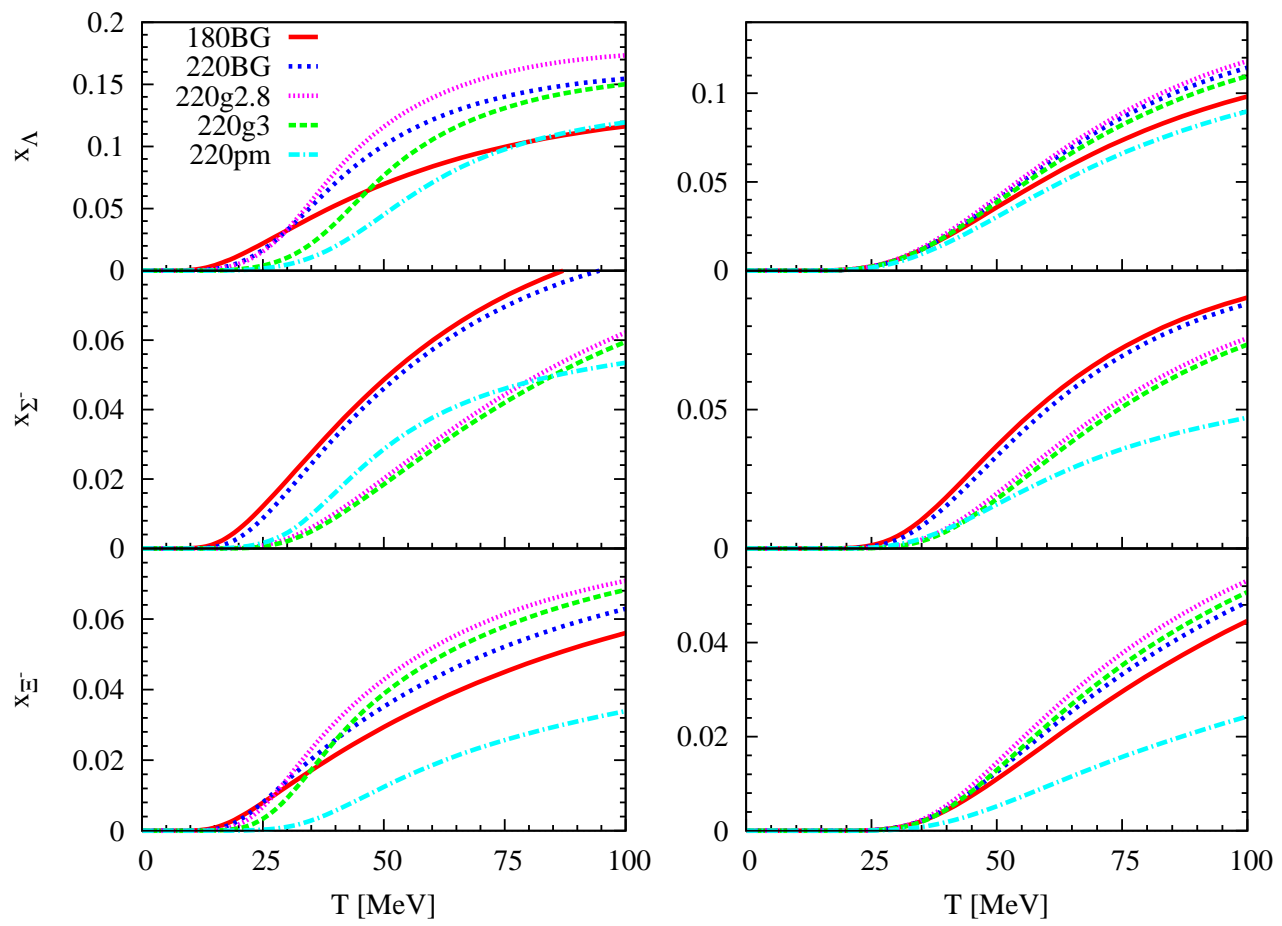

FIG. 7: (Color online) Same as Fig. 5 but as a function of temperature for $n_{B}=0.15 \mathrm{fm}^{-3}$ (right) and $n_{B}=0.3 \mathrm{fm}^{-3}$ (left) and an electron fraction of $Y_{e}=0.1$.
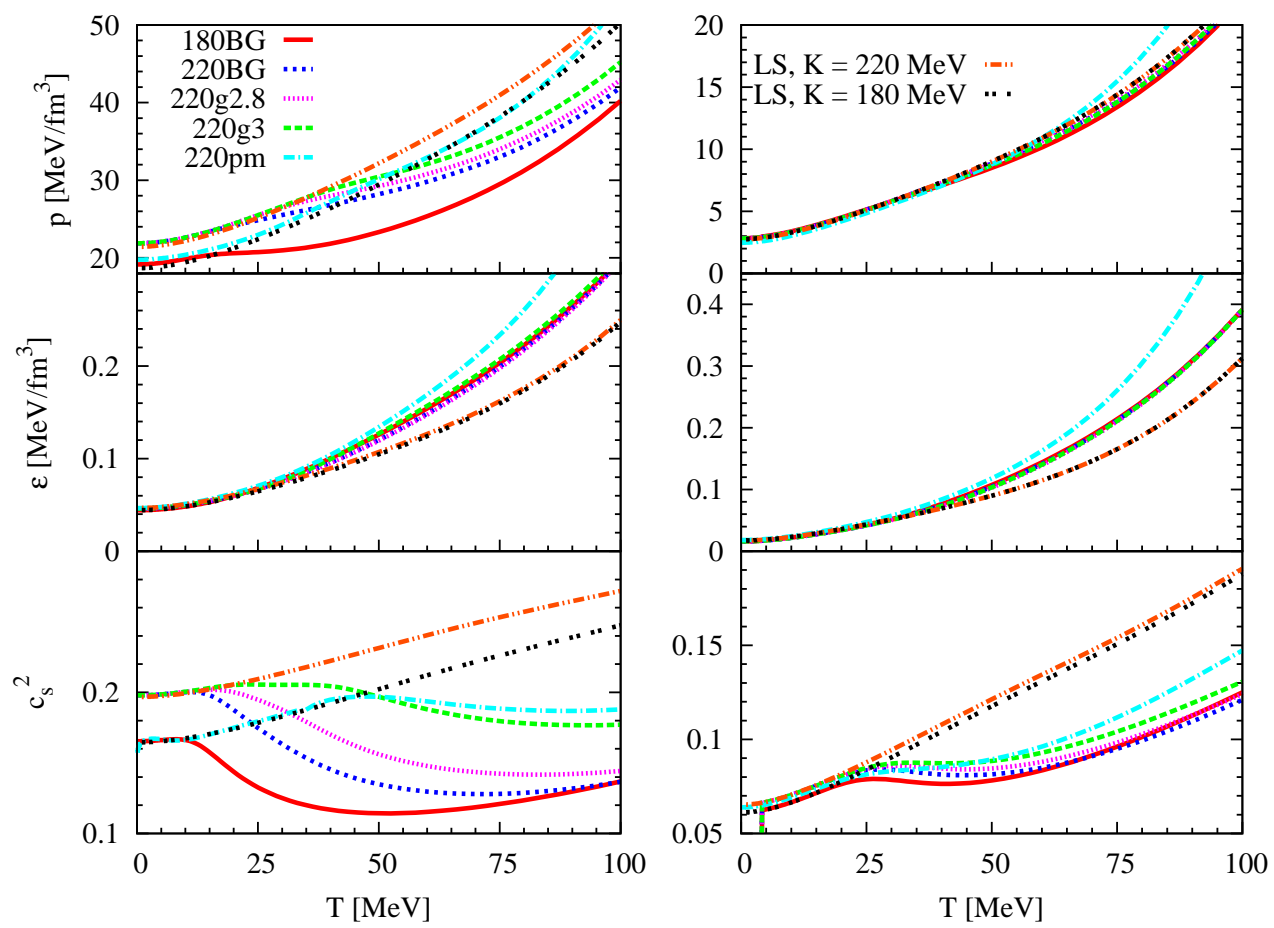

FIG. 8: (Color online) Same as Fig. 6, but as a function of temperature for $n_{B}=0.15 \mathrm{fm}^{-3}$ (right) and $n_{B}=0.3 \mathrm{fm}^{-3}$ (left) and an electron fraction of $Y_{e}=0.1$. 
explained and it is thus clear why the hyperon fractions are systematically lower for this EOS than for the others. Second, for the $\Sigma^{-}$-fraction, the two parametrizations by Balberg and Gal, 180BG and 220BG, clearly show a higher $\Sigma^{-}$-fraction than the other ones. The reason is again the strong $\Sigma^{-}$repulsion in the parametrizations $220 \mathrm{~g} 2.8$ and $220 \mathrm{~g} 3$.

At $n_{B}=0.3 \mathrm{fm}^{-3}$, hyperons appear at lower temperatures, depending on the EOS between roughly 15-25 $\mathrm{MeV}$. The differences between the EOS are more pronounced than at lower density. As mentioned earlier, this can be understood from the fact that, for sufficiently low density, due to thermal effects the kinetic energy should be dominant rendering the details of the interaction less important. For this higher density the $\Lambda$ hyperon is clearly the most abundant one, attaining between 10 and $17 \%$ at $T=100 \mathrm{MeV}$. The $\Sigma^{-}$-fraction at this temperature lies between 4 and $8 \%$ and for the $\Xi^{-}$, slightly less abundant, the fraction reaches $2-6 \%$.

The thermodynamic quantities, in particular pressure and sound speed clearly show the appearance of hyperons, inducing a softening in the EOS. For the lower density, the modifications in the pressure due to the presence of hyperons stays, however, relatively small up to $T=100 \mathrm{MeV}$, whereas $\epsilon$ and the sound speed show more important deviations between the purely nuclear case and the different cases with additional particles. Again the particle content of the EOS has more influence on the behavior of the thermodynamic quantities than the details of the interaction. This is, as already noticed before, not true at twice this density, where the different parametrizations give different results for pressure and sound speed. For the energy density the differences are smaller.

We recover most of the features discussed up to now as a function of density, too. This can be seen from Fig. 9, where the hyperon fractions are shown and from Fig. 10, where the thermodynamic quantities are displayed for $Y_{e}=0.1$. On the left panels $T=25 \mathrm{MeV}$, on the right panels $T=40 \mathrm{MeV}$. An interesting point which we have not seen before because the density has been too low is that at about $2.5 n_{0}$ a transition takes place, strongly increasing the hyperon fraction and with a strong effect on the thermodynamics. The $\Lambda$-fraction, for instance, can in certain models be larger than $30 \%$. The thermodynamic quantities, in particular the pressure, reflect this transition. A thorough discussion of the thermodynamics of this transition together with a detailed analysis of the stability is of order but beyond the scope of the present paper.

From the EOS alone we, of course, cannot answer the question whether the modifications in the thermodynamic quantities due to the presence of hyperons, pions and muons are relevant for the dynamics of a core collapse event or a neutron star merger. Here, we do not want to try to answer this question, but in order to get an idea we compare the pressure, energy density and sound speed profiles for a hot proto-neutron star. The data for this profile, thus $T, n_{B}, Y_{e}$, are shown on the right panels of Fig. 11 as a function of the radius. They are issued by a $1 \mathrm{D}$ simulation of the collapse of a $40 M_{\odot}$ progenitor with full Boltzmann neutrino transport employing the LS EOS with $K=180 \mathrm{MeV}$, see Ref. [79, 80], at about $400 \mathrm{~ms}$ after bounce. The left panels show the pressure, $\epsilon$ and the sound speed as a function of the radius for the different EOS corresponding to the given values of temperature, baryon density and electron fraction at this radius. Of course, this procedure does not give correct proto-neutron star profiles since these depend on the EOS. However, we think that with this remark of caution in mind, the comparison of the profiles is interesting and can give hints on the importance of the modifications in the high density and high temperature part of the EOS.

Let us first examine the data. The temperature is about $40 \mathrm{MeV}$ at the center, rising to more than 80 $\mathrm{MeV}$ at about $10 \mathrm{~km}$ from the center and decreasing then rapidly to a value between $5-10 \mathrm{MeV}$. The density is maximal at the center with a value slightly above $3 n_{0}$ decreasing to below saturation density at about $10 \mathrm{~km}$ from the center. $Y_{e}$ has a value of about 0.3 at the center. It decreases until about $15 \mathrm{~km}$, where the value is about 0.1 and rises then, reaching 0.5 at about $30 \mathrm{~km}$ from the center.

From the behavior of $T, n_{B}$ and $Y_{e}$ we would expect sensible modifications of the thermodynamic quantities only within a radius of about $15 \mathrm{~km}$ from the center of the proto-neutron star. This is indeed the case as can be seen from the left panels. Let us start with comparing the two different versions of the LS EOS. In the region very close to the center, up to roughly $8 \mathrm{~km}$, the pressure and the sound speed show differences between the two LS EOS. This is understandable since only in this region the density is high enough to allow for the differences in the nuclear interaction to play a significant role. Below saturation density at low temperatures the nuclear EOS is relatively well constrained so that in this region it would be surprising to see large differences. For high temperature and low density the kinetic part becomes dominant, so that no large differences due to the details of the nuclear interaction are to be expected. Therefore only the high density region remains where different nuclear EOSs can show very different behavior. Remember that we are not interested here in the details of the nuclear composition, which can have an influence on the thermodynamics and on the dynamics of a core collapse event too, see [5]. These occur mainly below saturation density and temperatures below $10 \mathrm{MeV}$, so that they cannot be resolved on the scales we are examining here.

Concerning the comparison with the extended EOS, we can see differences up to a radius of about $15 \mathrm{~km}$. These differences are in general more pronounced than those between the two version of the LS EOS. The pressure at the center varies between 60 and $100 \mathrm{MeV} / \mathrm{fm}^{3}$ from the "softest" to the "stiffest" EOS. The lowest pressure is obtained for 180BG, the second lowest for the LS EOS with $K=180 \mathrm{MeV}$ and all the others give values above 

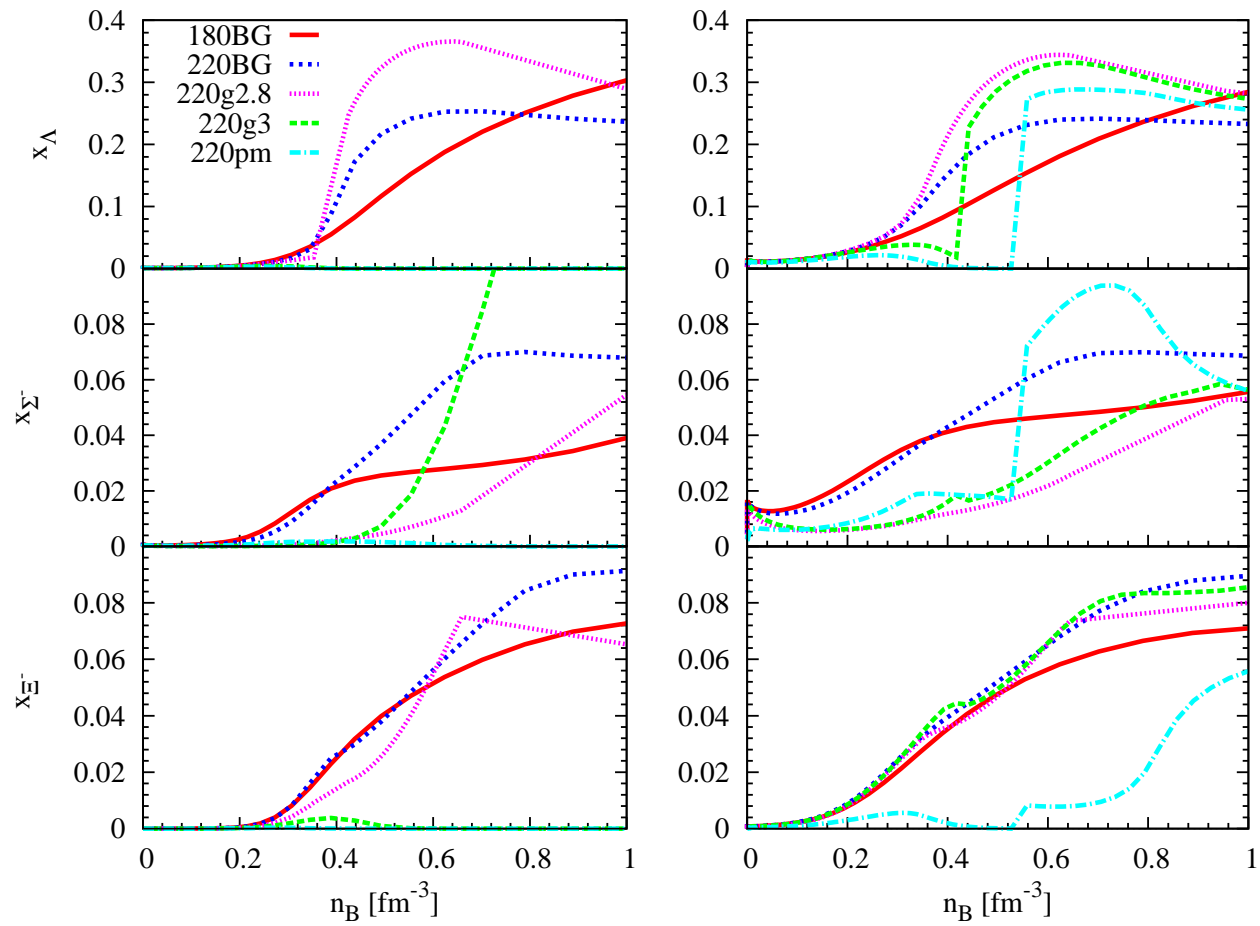

FIG. 9: (Color online) Same as Fig. 5 but as a function of baryon number density for an electron fraction $Y_{e}=0.1$ at a temperature of $25 \mathrm{MeV}$ (left) and $40 \mathrm{MeV}$ (right).
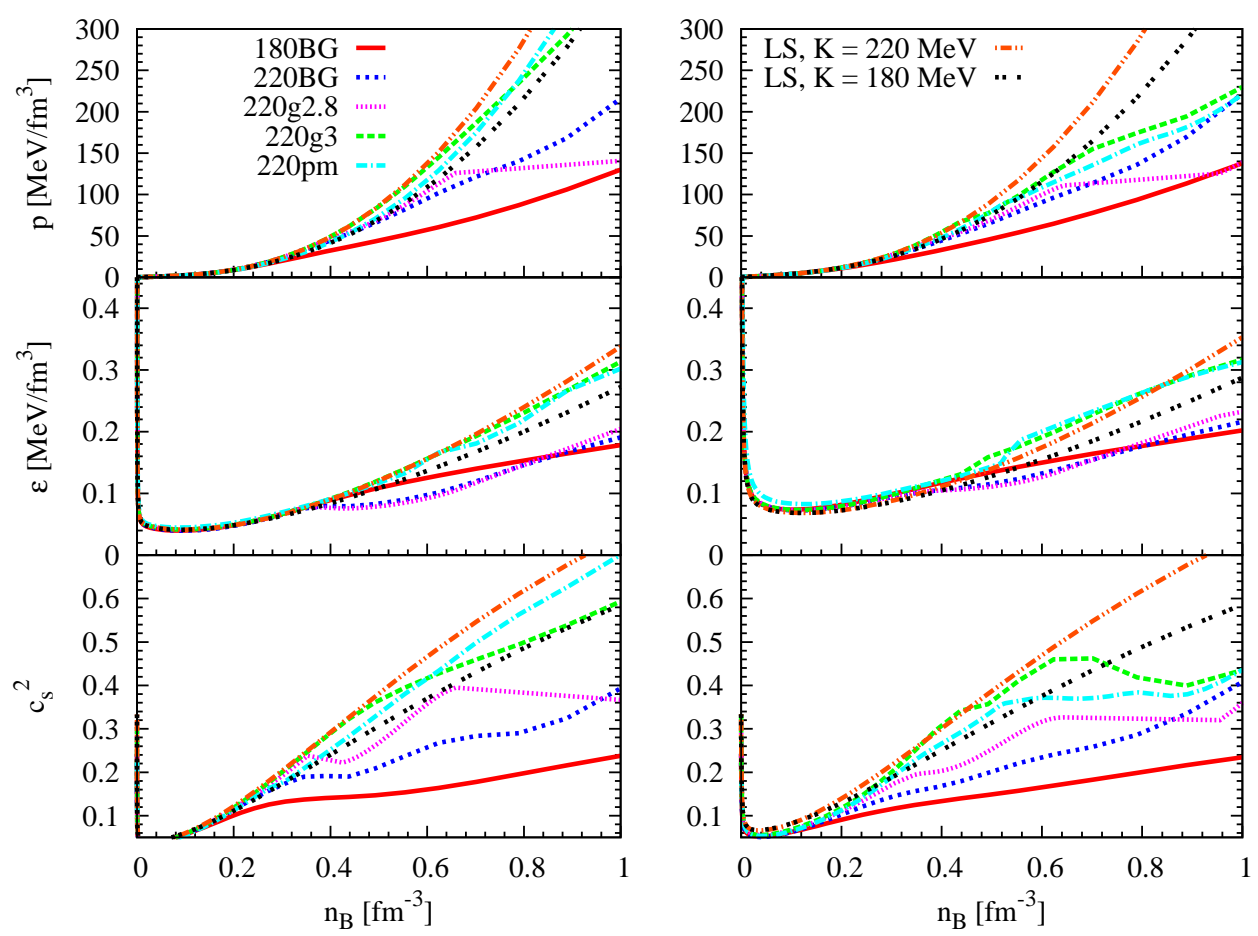

FIG. 10: (Color online) Same as Fig. 6 but as a function of baryon number density for an electron fraction $Y_{e}=0.1$ at a temperature of $25 \mathrm{MeV}$ (left) and $40 \mathrm{MeV}$ (right). 

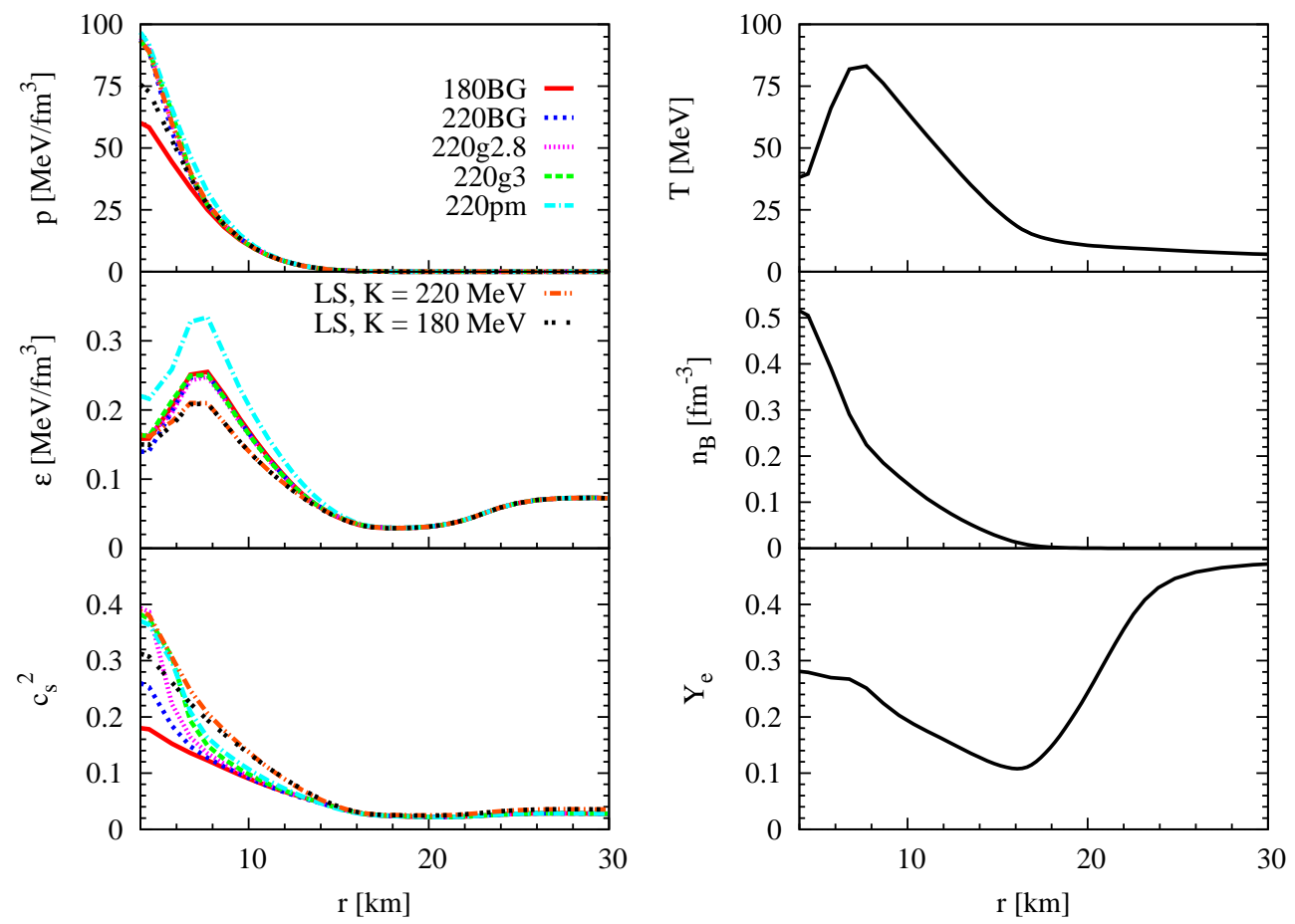

FIG. 11: (Color online) Pressure, energy density and sound speed (left) for different EOS as a function of radius for a protoneutron star profile (right) about $400 \mathrm{~ms}$ after bounce with a $40 M_{\odot}$ progenitor. Data (right) are from a simulation by M. Liebendörfer employing the LS EOS with $\mathrm{K}=180 \mathrm{MeV}$.

$90 \mathrm{MeV} / \mathrm{fm}^{3}$. This means that the influence of the additional particles on the pressure in this proto-neutron star is less important than the value of the nuclear incompressibility. We of course expect this conclusion to no longer hold if the central density, only about $3 n_{0}$ at the stage we are examining here, increases. And of course, as mentioned before, we have to be careful since we should recalculate the proto-neutron star profile with having a new EOS.

For $\epsilon$ we clearly have three groups of EOSs, the LS ones, those with hyperons and that with hyperons, pions and muons. The differences are most pronounced at the temperature maximum. This latter point is related to the fact that the energy density strongly depends on temperature. Moreover, the profile in $\epsilon$ closely follows the temperature profile. The previous discussion has already shown why $\epsilon$ has a distinct behavior depending on the particle content. The sound speed reflects the differences in pressure and energy density and can vary at the center between roughly $0.45 c$ and $0.6 c$.

The results for $220 \mathrm{pm}$ shown in Fig. 11 have again been calculated assuming the same chemical potential for electrons and muons. As discussed above, this represents probably an upper limit for the muonic effects on the EOS. Here, we have in principle the muon neutrino fraction at hand. Thus, assuming that the muon neutrinos are in thermal equilibrium, i.e. that they are described by a Fermi-Dirac distribution, we can determine the muon lepton chemical potential, and from this the muon chemical potential. We have computed the profiles with this muon chemical potential and, as expected, the differences are smaller, but the general trends are the same and in particular the results for $220 \mathrm{pm}$ are still very different from the other EOSs.

Up to now we have assumed that weak equilibrium with respect to strangeness is achieved. If, on the contrary, we assume that we have no weak strangeness changing reactions, which would correspond to having reaction timescales much longer than the hydrodynamic timescale of $10^{-6} \mathrm{~s}$, strangeness becomes a conserved quantum number. We do not consider this as a realistic scenario since the timescales estimated for the relevant processes are of the order of $10^{-6}$ or below, see e.g. [81]. We, however, find it instructive to compare our results with this extreme case. The hyperons have all negative strangeness, so that populating hyperonic states leads to a net negative strangeness. Typical production reactions for hyperons via the strong interaction, for example $n+n \rightarrow n+K+\Lambda$, are strangeness conserving and kaons are produced with positive strangeness. Thus kaons are the natural candidates for assuring vanishing net strangeness in thermal equilibrium. However, their mass of $m_{K} \approx 500 \mathrm{MeV}$ is rather high compared with the relevant chemical potentials and temperatures 
so that they are not very abundant ${ }^{3}$ if they are considered as an ideal gas. This in turn strongly suppresses the hyperon fractions and therefore the effects on the EOS compared with the more realistic scenario of strangeness changing weak equilibrium.

\section{SUMMARY}

At densities above roughly nuclear matter saturation and temperatures above several tens of $\mathrm{MeV}$, an equation of state based uniquely on nucleonic degrees of freedom and electrons is no longer realistic since many other states will appear. We have presented here an extended version of the LS EOS [3] including as additional particles hyperons, pions and muons intended to improve on the high density and high temperature part. For zerotemperature high density matter this question has already been studied for many years but up to now only very few work exists for finite temperature. The main problem in this type of exercise is that the interaction, which is already not well known for nucleons, is even less known for hyperons. We have adapted here a very simple phenomenological approach based on the hyperonic model of Balberg and Gal [46]. The parameters of the model have been readjusted in order to be compatible with available hyperonic data and in particular the observation of an almost two solar mass neutron star, PSR 1614-2230 [22]. Taking these constraints into account there still remains some freedom, so that we have discussed several parametrizations of the EOS in order to get an idea of the uncertainty. The ultimate goal should be, of course, to have a reliable microscopic approach to hyperonic matter compatible with data, but awaiting this step, we can, phenomenologically, study the effect of these additional particles on the thermodynamics of the system. The results show that key thermodynamic quantities as pressure, energy density and sound speed are influenced by the additional degrees of freedom in a nonnegligible way. The threshold temperature for the appearance of hyperons at saturation density lies at about $25 \mathrm{MeV}$, depending on the particular model applied. Due to the shift in the hadronic charge for a given electron fraction, muons seem to strongly influence the EOS in the regions where they become abundant.

We have concentrated here mainly on hyperons as additional particles. Nuclear resonances have not been considered for the moment. A study of this point is kept for future work. In another respect our EOS could be improved: we should not treat pions as a free gas but include interactions. At high temperatures this should not be very important, but for the low temperature and

\footnotetext{
3 Note that we are not discussing here possible medium modifications of the kaon properties which could lead to higher kaon abundances.
}

high density regime we expect it to have some influence.

The main application of our EOS should be astrophysical systems, first of all core collapse events of massive progenitors, collapsing eventually to a black hole. Neutron star mergers could be another application. We have demonstrated that our EOS can be successfully used in a numerical simulation of the collapse of a cold neutron star to a black hole. Of course the effect on realistic simulations including finite temperature has to be tested.

\section{Acknowledgments}

We would like to thank I. Vidaña for interesting discussions and M. Liebendörfer for providing us with the proto-neutron star data. This work has been partially funded by the SN2NS project ANR-10-BLAN-0503 and it has been supported by Compstar, a research networking programme of the European Science foundation. A.F.F. has been also supported by the Communauté française de Belgique - Actions de Recherche Concertées, and by the F.R.S.-FNRS (Belgium) via the contract of Chargé de Recherches.

\section{Appendix A: Some technical issues on the construction of the EOS tables}

Complete EOS tables, including the extended versions, will be prepared and made publicly available. In this Appendix we would like to discuss some technical issues encountered in the construction of those tables from the LS original routine. We remind that we have modified the original routine in two respects: (1) correcting the binding energy of the $\alpha$-particle (see Fig. 1); (2) extending the routine at densities $\lesssim 10^{-6} \mathrm{fm}^{-3}$, by extending the validity of the Maxwell and boundary construction files. This also permits to verify a correct matching with the low density EOS. However, some convergence issues at low temperatures and proton fractions or near critical temperature and density still remain. Convergence problems manifest in two ways: (1) no solution of the equilibrium equations, Eqs. (3.2) in Ref. [3], is found (especially at low temperature and electron fraction [31]), (2) the solution is discontinuous with respect to adjacent points in density, temperature, and/or electron fraction. In the latter case, the discontinuities appear as: (i) a rapid changes of the regime (with/without nuclei), which comes from a cross over the boundaries, (ii) a convergence towards a point far from the adjacent ones. The first kind of pathology might be due to the fact that a phase coexistence (and not a phase mixing) is considered to model the phase transition. The second one might be due to the sensitivity of the solution with respect to the starting point values in the minimization routine.

The construction of a table partially overcomes these discontinuities, since, when looking up the table, an interpolation is done and "critical" points in-between the 
grid points are usually avoided.

Another point to bear in mind when constructing the EOS table is the speed of sound. This is a crucial quantity in the hydrodynamic simulations, since it regulates the speed at which the information is propagated and it determines the time step in finite difference explicit schemes. Throughout the calculation, due to convergence problems, it might appear that the speed of sound is either less than zero or superluminal. This is of course non physical, so for those (rare) points we have recalculated the EOS replacing the value of the speed of sound with the one obtained in the case of a Fermi gas (in units of the speed of light) (see e.g. [82]):

$$
c_{s}^{2}=\frac{(\hbar c)^{2}}{3\left(m_{e} c^{2}\right)^{2}}\left(3 \pi n_{b} Y_{e}\right)^{2 / 3} .
$$

This replacement does of course not concern the region at high densities where the sound speed becomes superluminal due to the non-relativistic character of the LS EOS, see Section V A.

We have compared our tables to the O'Connor and Ott ones [83]. However, even if there is an agreement in the range where the LS routine is employed, it is not straightforward to make an exact comparison since the nuclear parameters that they employ are slightly different from the ones used in this paper.
[1] J. M. Lattimer and M. Prakash, Phys. Rept. 442, 109 (2007), astro-ph/0612440.

[2] W. Hillebrandt, K. Nomoto, and R. G. Wolff, Astronomy and Astrophysics 133, 175 (1984).

[3] J. M. Lattimer and F. D. Swesty, Nucl. Phys. A535, 331 (1991).

[4] H. Shen, H. Toki, K. Oyamatsu, and K. Sumiyoshi, Nucl. Phys. A637, 435 (1998), nucl-th/9805035.

[5] M. Hempel, T. Fischer, J. Schaffner-Bielich, and M. Liebendörfer (2011), 1108.0848.

[6] A. Burrows and J. M. Lattimer, Astrophys. J. 307, 178 (1986).

[7] S. I. Blinnikov, I. V. Panov, M. A. Rudzsky, and K. Sumiyoshi (2009), 0904.3849.

[8] S. Heckel, P. P. Schneider, and A. Sedrakian, Phys. Rev. C80, 015805 (2009), 0902.3539.

[9] M. Hempel and J. Schaffner-Bielich, Nucl. Phys. A837, 210 (2010), 0911.4073.

[10] G. Shen, C. J. Horowitz, and E. O'Connor, Phys. Rev. C83, 065808 (2011), 1103.5174.

[11] K. Sumiyoshi and G. Röpke, Phys. Rev. C77, 055804 (2008), 0801.0110.

[12] A. R. Raduta and F. Gulminelli, Phys. Rev. C82, 065801 (2010), 1009.2226.

[13] G. Shen, C. J. Horowitz, and S. Teige, Phys. Rev. C83, 035802 (2011), 1101.3715

[14] E. O'Connor, D. Gazit, C. J. Horowitz, A. Schwenk, and N. Barnea, Phys. Rev. C75, 055803 (2007), nuclth/0702044.

[15] A. Arcones et al., Phys. Rev. C78, 015806 (2008), 0805.3752.

[16] I. Sagert et al., Phys. Rev. Lett. 102, 081101 (2009), 0809.4225 .

[17] K. Sumiyoshi, C. Ishizuka, A. Ohnishi, S. Yamada, and H. Suzuki, Astrophys. J. Lett. 690, L43 (2009), 0811.4237.

[18] C. Ishizuka, A. Ohnishi, K. Tsubakihara, K. Sumiyoshi, and S. Yamada, J. Phys. G35, 085201 (2008), 0802.2318.

[19] H. Shen, H. Toki, K. Oyamatsu, and K. Sumiyoshi (2011), 1105.1666.

[20] K. Nakazato, K. Sumiyoshi, H. Suzuki, and S. Yamada, Phys. Rev. D81, 083009 (2010), 1004.0291.

[21] K. Nakazato, K. Sumiyoshi, and S. Yamada (2010),
1001.5084

[22] P. Demorest, T. Pennucci, S. Ransom, M. Roberts, and J. Hessels, Nature 467, 1081 (2010), 1010.5788.

[23] J. M. Lattimer and D. G. Ravenhall, Astrophys. J. 223, 314 (1978).

[24] J. M. Lattimer, C. J. Pethick, D. G. Ravenhall, and D. Q. Lamb, Nucl. Phys. A432, 646 (1985).

[25] J. Piekarewicz, J. Phys. G37, 064038 (2010), 0912.5103.

[26] J. M. Lattimer and F. D. Swesty, Lattimer-swesty eos web site, http://www.astro.sunysb.edu/dswesty/ lseos.html (1991-2012).

[27] M. Bender, P.-H. Heenen, and P.-G. Reinhard, Rev. Mod. PHys. 75, 121 (2003).

[28] P. Donati, P. M. Pizzochero, P. F. Bortignon, and R. A. Broglia, Phys. Rev. Lett. 72, 2835 (1994).

[29] A. F. Fantina, P. Donati, and P. M. Pizzochero, Phys. Lett. B676, 140 (2009), 0811.0456.

[30] R. Buras, M. Rampp, H. T. Janka, and K. Kifonidis, Astron. Astrophys. 447, 1049 (2006), astro-ph/0507135.

[31] J. M. Lattimer, Nuclear data needs for the equation of state in core collapse supernovae, http://www.physik. unibas.ch/ nic9sat/contrib/Lattimer_nicsat.pdf (2006).

[32] E. O'Connor and C. D. Ott, Class. Quant. Grav. 27, 114103 (2010), 0912.2393.

[33] F. X. Timmes and D. Arnett, Astrophys. J. Suppl. 125, 277 (1999).

[34] S. Weissenborn, D. Chatterjee, and J. Schaffner-Bielich (2011), 1111.6049.

[35] I. Bednarek, P. Haensel, J. L. Zdunik, M. Bejger, and R. Manka (2011), 1111.6942.

[36] J. R. Stone, P. A. M. Guichon, and A. W. Thomas, ArXiv e-prints (2010), 1012.2919.

[37] H. Chen, M. Baldo, G. F. Burgio, and H.-J. Schulze, Phys. Rev. D 84, 105023 (2011), 1107.2497.

[38] H.-J. Schulze and T. Rijken, Phys. Rev. C 84, 035801 (2011).

[39] G. F. Burgio, H.-J. Schulze, and A. Li, Phys. Rev. C 83, 025804 (2011), 1101.0726.

[40] E. Massot, J. Margueron, and G. Chanfray, ArXiv eprints (2012), 1201.2772.

[41] W. Keil and H. T. Janka, Astron. Astrophys. 296, 145 (1995). 
[42] T. W. Baumgarte, S. A. Teukolsky, S. L. Shapiro, H. T. Janka, and W. Keil, Astrophys. J. 468, 823 (1996).

[43] R. W. Mayle, J. R. Wilson, and M. Tavani, Astrophys. J. 418, 398 (1993).

[44] G. Raffelt and G. Sigl, Astropart. Phys. 1, 165 (1993), astro-ph/9209005.

[45] S. Hannestad, H. T. Janka, G. G. Raffelt, and G. Sigl, Phys. Rev. D62, 093021 (2000), astro-ph/9912242.

[46] S. Balberg and A. Gal, Nucl. Phys. A625, 435 (1997), nucl-th/9704013.

[47] H. Polinder, J. Haidenbauer, and U.-G. Meißner, Nucl. Phys. A779, 244 (2006), nucl-th/0605050.

[48] J. Haidenbauer, U.-G. Meißner, A. Nogga, and H. Polinder, Lect. Notes Phys. 724, 113 (2007), nucl-th/0702015.

[49] S. R. Beane et al. (NPLQCD), Nucl. Phys. A794, 62 (2007), hep-lat/0612026.

[50] I. Vidaña, A. Polls, A. Ramos, and H. J. Schulze, Phys. Rev. C64, 044301 (2001).

[51] H. J. Schulze, M. Baldo, U. Lombardo, J. Cugnon, and A. Lejeune, Phys. Rev. C57, 704 (1998).

[52] T. A. Rijken, V. G. J. Stoks, and Y. Yamamoto, Phys. Rev. C59, 21 (1999), nucl-th/9807082.

[53] H. Djapo, B.-J. Schaefer, and J. Wambach, Eur. Phys. J. A36, 101 (2008), 0802.2646.

[54] I. Vidaña, D. Logoteta, C. Providencia, A. Polls, and I. Bombaci, Europhys. Lett. 94, 11002 (2011), 1006.5660.

[55] P. K. Saha et al., Phys. Rev. C70, 044613 (2004), nuclex/0405031.

[56] T. Nagae et al., Phys. Rev. Lett. 80, 1605 (1998).

[57] M. Kohno, Y. Fujiwara, Y. Watanabe, K. Ogata, and M. Kawai, Phys. Rev. C74, 064613 (2006), nuclth/0611080.

[58] P. Khaustov et al. (AGS E885), Phys. Rev. C61, 054603 (2000), nucl-ex/9912007.

[59] K. Nakazawa (KEK-E176 and J-PARC-E07), Nucl. Phys. A835, 207 (2010).

[60] M. Baldo, G. F. Burgio, and H. J. Schulze, Phys. Rev. C61, 055801 (2000), nucl-th/9912066.

[61] I. Vidaña, A. Polls, A. Ramos, L. Engvik, and M. Hjorth-Jensen, Phys. Rev. C62, 035801 (2000), nuclth/0004031.

[62] H. Djapo, B.-J. Schaefer, and J. Wambach, Phys. Rev. C81, 035803 (2010), 0811.2939.

[63] S. Weissenborn, I. Sagert, G. Pagliara, M. Hempel, and J. Schaffner-Bielich, Astrophys. J. 740, L14 (2011),
1102.2869 .

[64] M. Alford et al., Nature 445, E7 (2007), astro$\mathrm{ph} / 0606524$.

[65] S. Weissenborn, D. Chatterjee, and J. Schaffner-Bielich (2011), 1112.0234.

[66] F. Hofmann, C. Keil, and H. Lenske, Phys.Rev. C64, 025804 (2001), nucl-th/0008038.

[67] L. Bonanno and A. Sedrakian (2011), 1108.0559.

[68] E. Gourgoulhon, Astron. Astrophys. 252, 651 (1991).

[69] A. Chodos, R. L. Jaffe, K. Johnson, C. B. Thorn, and V. F. Weisskopf, Phys. Rev. D9, 3471 (1974).

[70] H. Dimmelmeier, J. Novak, J. A. Font, J. M. Ibáñez, and E. Müller, Phys. Rev. D 71, 1 (2005).

[71] J. A. Font, Living Rev. in Relativity 11 (2008), URL http://www .livingreviews .org/lrr-2008-7.

[72] P. Grandclément and J. Novak, Living Rev. Relativity 12, 1 (2009), http://www.livingreviews.org/ lrr-2009-1.

[73] L.-M. Lin and J. Novak, Class. Quantum Grav. 23, 4545 (2006).

[74] E. Gourgoulhon, P. Grandclément, J.-A. Marck, and J. Novak, LORENE: Langage Objet pour la RElativité NumériquE, http://www. lorene.obspm.fr (1997-2012).

[75] I. Cordero-Carrión, P. Cerdá-Durán, H. Dimmelmeier, J. L. Jaramillo, J. Novak, and E. Gourgoulhon, Phys. Rev. D 79, 1 (2009), 0809.2325.

[76] L.-M. Lin and J. Novak, Class. Quantum Grav. 24, 2665 (2007).

[77] M. D. Duez, S. L. Shapiro, and H.-J. Yo, Phys. Rev. D 69, 104016 (2004), arXiv:gr-qc/0401076.

[78] A. Perego, Private communication.

[79] M. Liebendörfer, O. E. B. Messer, A. Mezzacappa, S. W. Bruenn, C. Y. Cardall, and F.-K. Thielemann, Astrophysical Journal Supplement 150, 263 (2004), arXiv:astro-ph/0207036.

[80] T. Fischer, S. C. Whitehouse, A. Mezzacappa, F. K. Thielemann, and M. Liebendörfer, Astronomy and Astrophysics 499, 1 (2009), 0809.5129.

[81] G. E. Brown, V. Thorsson, K. Kubodera, and M. Rho, Phys. Lett. B291, 355 (1992).

[82] E. Chabanat, J. Meyer, P. Bonche, R. Schaeffer, and P. Haensel, Nucl. Phys. A627, 710 (1997).

[83] E. O'Connor and C. D. Ott, stellarcollapse.org, http: //www.stellarcollapse.org (2008-2012). 